\title{
Anabases
}

ANABASES Traditions et réceptions de l'Antiquité

$31 \mid 2020$

Varia

\section{Guillaume BUDÉ, De asse et partibus eius. L'As et ses fractions. Livres I-III}

\section{Laure Hermand-Schebat}

\section{OpenEdition}

\section{Journals}

Édition électronique

URL : https://journals.openedition.org/anabases/10897

DOI : 10.4000/anabases. 10897

ISSN : 2256-9421

\section{Éditeur}

E.R.A.S.M.E.

\section{Édition imprimée}

Date de publication : 27 juin 2020

Pagination : 227-228

ISSN : 1774-4296

\section{Référence électronique}

Laure Hermand-Schebat, "Guillaume BuDÉ, De asse et partibus eius. L'As et ses fractions. Livres I-III », Anabases [En ligne], 31 | 2020, mis en ligne le 27 juin 2020, consulté le 17 novembre 2022. URL : http:// journals.openedition.org/anabases/10897 ; DOI : https://doi.org/10.4000/anabases.10897 

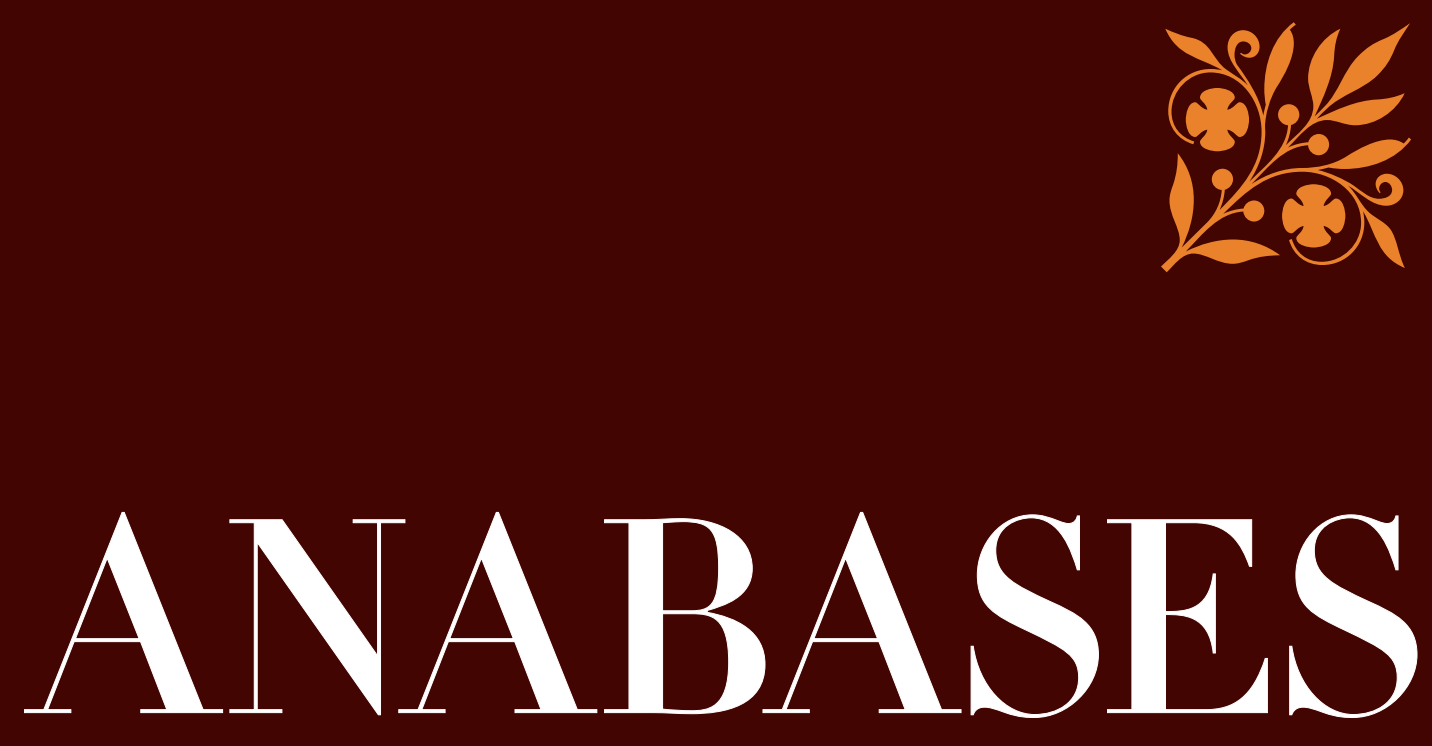

Traditions et Réceptions de l'Antiquité

\section{No31 \\ 2020}

Deux jours à Cherchel La France au miroir de l'Antique Cing lettres du père Festugière à Michel Foucault La réception des Arithmétiques de Diophante L'apport scientifique des bases de données 
ANABASES

Traditions et Réceptions de l'Antiquité

Revue de l'équipe de recherche E.R.A.S.M.E.

Université Toulouse-Jean Jaurès (UT2J)

Anabases dispose d'un Comité de lecture international. Chaque article envoyé à la rédaction est soumis, une fois anonymisé, à l'expertise de deux spécialistes qui rendent un rapport écrit. Les deux rapports anonymisés sont transmis à l'auteur qui tient compte des observations en vue de la publication.

\section{Comité SCIENTIFique}

Germaine Aujac (université Toulouse-Jean Jaurès : histoire de la géographie et des sciences antiques)

Florence Bouchet (université Toulouse-Jean Jaurès : littérature médiévale)

Hinnerk Brunns (CNRS : histoire économique et sociale ancienne et contemporaine)

Paulo Butti de Lima (université de Bari : historiographie et réception de l'Antiquité)

Luciano CANFora (université de Bari : littérature et histoire anciennes, historiographie)

Giovanna Ceserani (Stanford University : histoire intellectuelle et historiographie de la tradition classique)

Temístocles Cezar (université de Porto Alegre : historiographie moderne)

Serafina Сuомо (University of London, Birkbeck College : histoire des mathématiques et des sciences)

Paul Demont (université de Paris Sorbonne : philologie grecque et héritage classique)

Marie-Laurence Desclos (université de Grenoble II : philosophie de l'Antiquité)

Olivier Devillers (université de Bordeaux 3 - Michel-de-Montaigne : littérature et historiographie latines)

Andrea Giardina (Istituto italiano di scienze umane : histoire du monde romain et de ses réceptions)

Ève Gran-Aymerich (AIBL : histoire de l'archéologie et des transferts culturels)

François Hartog (EHEss : historiographie ancienne et moderne)

Geneviève Hoffmann (université de Picardie : histoire des mondes grecs)

Christian JACOB (CNRS/EHESS : histoire comparée et épistémologie des savoirs)

Suzanne Marchand (Louisiana State University : histoire du classicisme et de l'orientalisme)

Wilfried NIPPEL (Humboldt Universität Berlin : histoire et historiographie de l'Antiquité)

Sylvie Pitria (université de Paris I-Panthéon Sorbonne : histoire et historiographie du monde romain)

Stéphane Ratтi (université de Franche-Comté - Besançon : philologie et héritage latin)

Comité de RÉdACtion

Clément Bertau-Courbières, Corinne Bonnet, Laurent bricault, Clément Bur, Adeline Grand-Clément, Anne-Hélène Klinger-Dollé, Véronique Krings, Thibaud Lanfranchi, Pascal Payen, Grégory Reimond, Sarah Rey, Catherine Valenti, Noémie Villacèoue

Éditeur RESPONSABLE

Clément Bur

Éditrice ADJOINTE

Catherine VALENTI

Sites WEB

http://plh.univ-tlse2.fr

Revues.org : http://anabases.revues.org

Aвonnement et vente aU numéro

Éditions De Boccard - 4, rue de Lanneau - 75005 Paris

info@deboccard.com - www.deboccard.com

Tél. : 0033/(0)143260037 - Fax : 0033/(0)143548583 




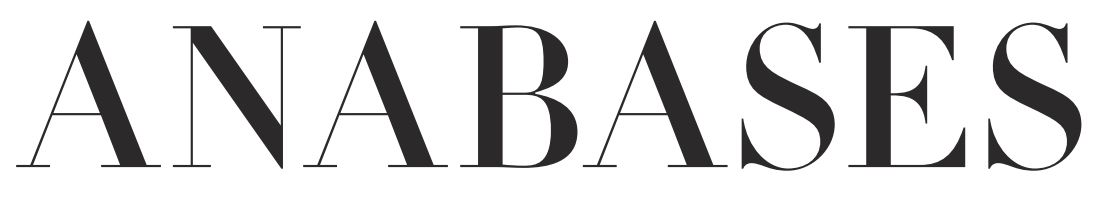

Traditions et Réceptions de l'Antiquité

$$
\begin{aligned}
& N \circ 31 \\
& 2020
\end{aligned}
$$

E.R.A.S.M.E.

Université Toulouse - Jean Jaurès 



\section{Sommaire}

$\mathrm{N}^{\circ} 31-2020$

\section{Traditions du patrimoine antique}

Markus Stachon

Odious and Yet Lovely: Carl Orff's Scenic Cantata Catulli Carmina. . . . I I

Jean-François Domenget

“ Deux jours à Cherchel » de Montherlant, commentaire d'un manuscrit inédit . . . . . . . . . . . . . . 25

Dossier coordonné par Arnaud Saura-Ziegelmeyer

"La France au miroir de l'Antique » . . . . . . . . . . . . . . . 43

Ludivine Beaurin et Laurent Bricault

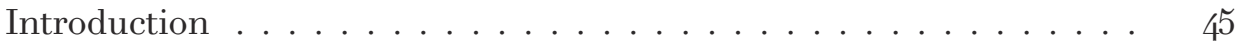

Marie-Laurence HaAck et Martin Miller

Une Antiquité sélective - Eine selektive Antike.

La disparition des Étrusques dans les manuels français et italiens du

$\mathrm{xx}^{\mathrm{e}}$ siècle. Das Verschwinden der Etrusker aus den deutschen

Schulbüchern des 20. Jahrhunderts . . . . . . . . . . . . . . . . . . .

Ludivine BEAURIN

Étudier l'Égypte ancienne à l'école : acquisition d'un savoir

et/ou construction d'une image mentale? . . . . . . . . . . . . . . 69

Aurélie Rodes

Du bon usage des manuels scolaires pour étudier la réception

des Gaulois dans l'enseignement secondaire. . . . . . . . . . . . . . . .

Guillaume Diana

Les langues et cultures de l'Antiquité dans la réforme du Collège de 2016 : une volonté absolue d'interdisciplinarité . . . . . . . . . . . . . 99

Corinne Bonnet

En guise de conclusion : pour une histoire des savoirs scolaires . . . . . II9 


\section{Archéologie des savoirs}

Pierre Vesperini

“ Un gentil mécréant, avec qui l'on entre aussitôt dans le seul monde qui compte »Cinq lettres du père Festugière

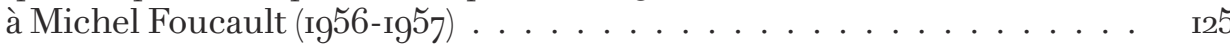

Odile Le Guillou - Kouteynikoff

La réception des Arithmétiques de Diophante par Guillaume Gosselin

de Caen, algébriste de la Renaissance française . . . . . . . . . . . . I3

\section{Actualités et débats}

Tiphaine Annabelle Besnard

L’ “ Incroyable » trésor de Damien Hirst présenté à Venise en 20I7 . . . . .

\section{Lire, relire la bibliothèque des sciences de l'Antiquité}

Guillaume de Méritens de Villeneuve

Relire “ The Allegiance of Labienus » $(J R S, 28$, I938) et dépasser

l'inertie historiographique autour des travaux de Ronald Syme . . . . . .

\section{L'atelier de l'histoire : les chantiers historiographiques}

Actualité du théâtre antique » (coordonné par Malika Bastin-Hammou) $\left(n^{\circ} 5\right)$

Claire Lechevalier

La génération sacrifiée des enfants des Atrides. Électre/Oreste

mis en scène par Ivo Van Hove à la Comédie-Française . . . . . . . . . . .

Les mots de l'Antiquité (coordonné par Magali Soulatges) $\left(n^{\circ} 11\right)$

Florent LiBRAL

Conjurer le spectre d'Épicure ? Noms et définitions de l'atome

dans la philosophie d'Emmanuel Maignan (I6oI-ı676)

Antiquités numériques (coordonné par Elodie Guillon) ( $\left.n^{\circ} 2\right)$

Adeline Grand-Clément et Élodie GuILlon

L'apport scientifique des bases de données : réflexion croisée sur

les programmes MAP (Mapping Ancient Polytheisms) et Eurykleia . . . . I98

Droit et réception de l'Antiquité (coordonné par Marielle de Béchillon) ( ${ }^{\circ} 7$ )

Marielle DE BÉchILlon

Lettre ouverte aux juristes désirant étudier la causalité et

la responsabilité dans l'Antiquité grecque. Autour du dossier

“ Aitia, causalité juridique, causalité philosophique » . . . . . . . . . 
Atelier des doctorants

Grégory Reimond

Historia de la arqueología y biografía intelectual,

o la mirada (in)discreta del historiador-voyeur . . . . . . . . . . . 2IO

\section{Comptes rendus}

John Boardman, Julia Kagan et Claudia Wagner

avec la contribution de Catherine Phillips, Natter's Museum Britannicum :

British gem collection and collectors of the mid-eighteenth century

(Thibault Girard) .................................... 223

Lisbeth Bredholt Christensen et Jesper Tae Jensen (éds.)

Religion and Material Culture: Studying Religion and Religious Elements

on the Basis of Objects, Architecture and Space (Irene Berti) . .......... 224

Guillaume BudÉ

De asse et partibus eius. L'As et ses fractions. Livres I-III,

éd. et trad. L.-A. SANchi (Laure Hermand-Schebat) . . . . . . . . . . . . . . 227

Maurizio Campanelli et Frances Muecke (éds.)

The Invention of Rome : Biondo Flavio's Roma Triumphans and Its Worlds

(Cyrielle Landrea) ...................................... 228

Jean-Noël Castorio

Rome réinventée. L'Antiquité dans l'imaginaire occidental de Titien à Fellini

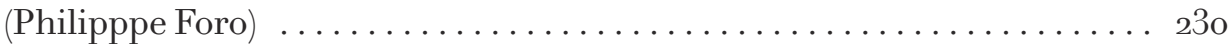

Ettore CinNELLA

Lo zar e il latino. Gli studi classici in Russia tra Otto e Novecento

(Pier Giuseppe Michelotto) .............................. 23I

Bianca De Divitiıs, Fulvio Lenzo et Lorenzo Miletti (éds.)

Ambrogio Leone's De Nola, Venice I5I4. Humanism and Antiquarian

Culture in Renaissance Southern Italy (Clarisse Evrard) . . . . . . . . . . . 234

Michael A. Flower (éd.)

The Cambridge Companion to Xenophon (Olivier Gengler) ............ 235

Rebecca Futo Kennedy (dir.)

Brill's Companion to the Reception of Eschylus

(Dominique Millet-Gérard) . ................................. 237 
Antonio Gonzales et Maria Teresa Schettino (dir.)

Les sons du pouvoir des autres. Actes du troisième colloque SoPHiA, 27-28 mars 2014 (Clément Bur) . . . . . . . . . . . . . . . . . . . 238

Christa Gray, Andrea Balbo, Richard M. A. Marshall et Catherine E. W. Steed (éds.)

Reading Republican Oratory. Reconstructions, Contexts, Receptions (Cyrielle Landrea) . . . . . . . . . . . . . . . . . . . . . . . 240

Tanja Itgenshorst et Philippe Le Doze (dir.), La norme sous la république et le Haut-Empire romains.

Élaboration, diffusion et contournements (Clément Bur) . . . . . . . . 242

Hans Kopp et Christian Wendt (éds.)

Thalassokratographie. Rezeption und Transformation antiker

Seeherrschaft (Hinnerk Bruhns) ............................ 244

Antonio La Penna

Io e l'antico. Conversazione con Arnaldo Marcone (Federico Santangelo) . . 2446

Aude Lehmann (dir.)

Diderot et l'Antiquité classique (Odile Richard-Pauchet) . . . . . . . . . . 247

Élise LeHoux,

Mythologie de papier. Donner à voir l'antiquité entre France et Allemagne

(xvIII siècle-milieu du xIX $x^{e}$ siècle), préface de François Lissarrague

(Geneviève Hoffman) . . . . . . . . . . . . . . . . . . . . . . . . 25I

Dino Piovan

Tucidide in Europa. Storici e storiografia greca nell'età dello storicismo, postface de Ugo Fantasia (Luca Iori) . . . . . . . . . . . . . . . . 252

Sylvie Rougier-Blanc (éd.)

Athénée de Naucratis. Le banquet des savants, livre XIV : Spectacles, chansons, danses, musiques et desserts (texte, traduction et notes - études et travaux) (Arnaud Saura-Ziegelmeyer) . . . . . . . . . . . . . . . . 254

Isabelle TORRANCE

Euripides (Alexia Dedieu) . . . . . . . . . . . . . . . . 256

Résumés . . . . . . . . . . . . . . . . . . . . . . . . . . 249

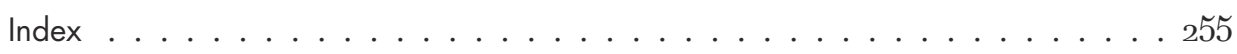




\section{Comptes rendus de lecture}



John Boardman, Julia Kagan et Claudia

WAGNER, avec la contribution de

Catherine Phillips, Natter's Museum

Britannicum: British gem collection and collectors of the mid-eighteenth century, Oxford, Archaeopress, 20I7, iv+3o4p., $£ 55$ / ISBN 978I784917272.

J. Boardman, J. Kagan, C. Wagner et C. Philips, en collaboration avec la maison d'éditions Archaeopress et le musée de l'Ermitage, proposent ici un beau livre, très richement illustré de nombreuses photographies en couleurs. Glissé à l'intérieur d'une élégante jaquette, l'ouvrage relié est agréable à lire, dans un style simple et fluide, comme les auteurs nous y ont habitués. Sauf exception, les illustrations sont de bonne qualité et de taille respectable, dans une mise en page soignée bien qu'aléatoire par endroits (décalage entre le commentaire sur une page et les images sur l'autre; juxtaposition des images parfois collées les unes aux autres, parfois non; manque d'uniformité éditoriale). Mises à part ces quelques broutilles, c'est un bel objet qui honore la mémoire d'O. Neverov, à qui il est dédié.

Onze chapitres organisés en deux parties sont nécessaires pour décortiquer le magnum opus de Lorenz Natter, miraculeusement retrouvé dans les archives du musée de l'Ermitage dans les années i97o. La première partie retrace la biographie de Lorenz Natter, graveur, collectionneur de gemmes et d'intailles, fin historien de l'art, avec une attention particulière sur son parcours en Grande-Bretagne et en
Russie (chapitres II, III et IV). La destinée de son manuscrit est mise en lumière dans les chapitres I, V et VI. En une trentaine de pages, tout est dit. Une bibliographie (p. 33-39) vient clore cette courte première partie, qui replace Lorenz Natter comme l'un des piliers des études sur la glyptique antique du XviıI ${ }^{\mathrm{e}}$ siècle.

Avec la seconde partie, on entre dans le vif du sujet. Pas moins de 250 pages la constituent (p. 40-294). Les auteurs ont décortiqué les manuscrits de Lorenz Natter pour identifier l'original de chaque dessin (chapitre VII). La description de Lorenz Natter (en ancien français) - lorsqu'elle existe - a été replacée en vis-à-vis du commentaire moderne (en anglais), suivi d'une bibliographie succincte, qui est réduite dans la plupart des cas à la référence fondamentale et au numéro d'inventaire. En tout, plus de 53o notices ont été établies. Ce travail est agrémenté de reproductions de pages entières d'index établis par Lorenz Natter lui-même (chapitre X) et une cinquantaine de planches de dessins à partir desquelles on peut juger de son extraordinaire talent de dessinateur (chapitre XI). Le volume se conclut par une série d'index et quelques références bibliographiques abrégées propres au catalogue.

Outre le catalogue qui, en soi, ne laisse guère de place à une critique autre que formelle, ce sont les chapitres VIII et IX sur l'histoire des collections et des collectionneurs anglais qui ancrent le manuscrit dans son contexte. Après les gemmes du catalogue, ce sont donc les 
collectionneurs qui sont présentés dans le chapitre VIII. Pour chacun, une courte biographie est proposée, toujours en lien avec leurs goûts en matière d'antiques et le catalogue de Lorenz Natter. L'accent est aussi porté sur la propre collection de Lorenz Natter (chap. IX) : I37 pierres, datant $\mathrm{du} \mathrm{II}^{\mathrm{e}}$ s. av. J.-C au XviI ${ }^{\mathrm{e}}$ s., mêlées au catalogue, dont 49 ont aujourd'hui disparu. Riche, non par la quantité mais par la diversité et la qualité des gravures, sa collection est le reflet de sa passion pour la glyptique tant comme artisan-graveur que comme amateur éclairé, et les descriptions qu'il donne de chaque pierre, l'expression vivante de cette passion. On explore là la sensibilité du collectionneur. Grâce à ces deux chapitres qu'il convient de relier à la première partie, le catalogue - à première vue un peu aride - prend une véritable dimension historiographique ainsi qu'une teinte biographique insoupçonnée. In fine, l'ouvrage offre une large vue sur la réception de l'Antiquité en Angleterre au Xvır' siècle par le truchement de la personnalité de Lorenz Natter.

À ces qualités indéniables, quelques regrets tout au plus peuvent être formulés. En premier lieu, si l'on constate que Lorenz Natter avait bien compris que le sens de lecture des intailles est celui du moulage, on aurait souhaité que les moulages des intailles photographiées soient reproduits. Les dessins de Lorenz Natter, même si exécutés avec finesse et habileté, ne sont pas aussi fidèles qu'une photographie. De surcroît l'image en creux ne permet pas toujours de distinguer tous les détails. Le fastidieux travail d'identification des gemmes aurait mérité cet effort supplémentaire. La dimension des dessins - au minimum aurait également été un plus. En second lieu, on aurait attendu, pour chaque entrée, une bibliographie plus complète que celle proposée. Chaque œuvre, depuis Lorenz Natter jusqu'à nos jours, aurait bénéficié de cette mise en perspective tant scientifique qu'historiographique. Pour ne prendre que les pierres figurant Diomède et le rapt du Palladion (cat. $\mathrm{n}^{\text {os }} 236$, 25I et 48I), n'aurait-il pas été utile aux lecteurs de renvoyer à l'ouvrage de J.-M. Moret (Les pierres gravées représentant le rapt du Palladion) paru en i997? Le commentaire complet et la bibliographie exhaustive proposés par J.-M. Moret pour la pierre du château d'Alnwick en particulier (cat $\mathrm{n}^{\circ} 25 \mathrm{I}$ de Natter; cat. $\mathrm{n}^{\circ} 223$ de Moret) auraient ouvert une porte sur l'iconographie du thème. Les références fondamentales - certes - mais anciennes à J. Tassie ou à L. de Gravelle sont plus hermétiques ; l'article d'O. Neverov et J. Kagan peut-être plus anecdotique que la monographie de Moret. La remarque est valable également pour les gemmes figurant Sérapis : la thèse de R. Veymiers est désormais incontournable...

Tout cela n'enlève rien à ce beau livre. C'est une publication de premier ordre qui s'inscrit dans le sillage des travaux édités (papier et online) par le Classical Art Research Center de l'Université d'Oxford, à la croisée des chemins entre l'histoire de l'art antique, post-antique et la réception de ces images à l'époque moderne.

Thibault GIRARD École normale supérieure de Lyon thibault.girard@ens-lyon.fr

\section{Lisbeth Bredholt Christensen et Jesper TAe Jensen (éds.), Religion and Material Culture: Studying Religion and Religious Elements on the Basis of Objects, Architecture and Space, Turnhout, Brepols Publishers, 20I7, 378 p., Io5€/ ISBN 9782503569ooo.}

Il volume raccoglie gli atti di una conferenza tenutasi nel 20II al Centre for Bible and Cultural Memory di Copenhagen e al National Museum of Denmark. La novità della pubblicazione è nell'affrontare attraverso un approccio 
materiale non solo il tema del culto (su cui già esiste vastissima letteratura), ma più in generale della religione, intesa anche nei suoi aspetti teologici, astratti e discorsivi. Intenzionalmente, la raccolta non si limita ad analizzare un solo ambito culturale o cronologico, ma al contrario i contributi spaziano dalla preistoria all'età contemporanea, affiancando realtà culturalmente e geograficamente lontane, come le culture del Bronzo scandinavo, il Neolitico vicino-orientale e la commemorazione delle vittime dell'Olocausto. L'intenzione è quella di testare una definizione possibilmente universale di religione, basata sia sulla cultura materiale che sui testi, che trascenda le diversità storiche e culturali (p. I7).

Il volume si articola in tre sezioni tematiche: "Methodology", "Archaeology" e "Text". Nella prima sezione, Lisbeth Bredholt Christensen mette in discussione le definizioni tradizionalmente proposte per lo studio di religioni che si basano su testi sacri (Cristianesimo, Islam) e indica le coordinate teoriche per un approccio alla storia della religione che parta dal materiale, piuttosto che dai modelli. Le definizioni tradizionali incentrate sugli aspetti ideologici, soprannaturali e trascendenti, adatte allo studio della religione come pratica discorsiva e sistema di valori, sono efficaci quando si lavora con i testi, ma applicate alla cultura materiale preistorica sono di scarsa utilità. I nuovi approcci basati sulla neurobiologia e sull'assunto dei "Material Culture Studies" che la materialità (immagini, oggetti, spazi, ma anche movimenti e danze), non solo esprime emozioni e pensieri, ma anche genera, orienta e condiziona questi ultimi, aprono nuove possibilità di indagine. Decisivo è, nel pensiero dell'autrice, il ruolo dell'autorità e delle istituzioni, senza le quali non è possibile parlare di religione, sebbene possano certamente esistere fenomeni di spiritualità. L'importanza della cultura materiale religiosa è ribadita da Morten Warmind ("even a god with no material being requires a cultic focus", p. 59), mentre David A. Warburton considera l'apporto della cultura materiale alla storia del pensiero, partendo dalla concezione che il pensiero astratto e discorsivo non si sia sviluppato prima dell'età del Bronzo nel Vicino Oriente. La sua conclusione è che, se da una parte la cultura materiale è la precondizione necessaria per lo sviluppo del pensiero astratto, dall'altra l'invenzione della scrittura ha giocato un ruolo essenziale nello sviluppo della capacità di esprimere il pensiero; di conseguenza, le moderne definizioni di religione (basate sul testo) sono adeguate, ma solo nel senso che ciò che noi oggi chiamiamo religione, non esisteva prima (p. 64). La ricca sezione dedicata all'archeologia si apre con il contributo di Emmanuel Anati, che considera la religione soprattutto dal punto di vista della pratica cultuale, giungendo alla conclusione, opposta a quella di Warburton, che la religione possa considerarsi una delle primissime manifestazioni di vita spirituale dell'essere umano. Pratiche cultuali legate ai riti di sepoltura sono attestate infatti già per il Paleolitico: in particolare l'arte pittorica e i graffiti sulle rocce attestano l'esistenza di un culto degli antenati. La spiritualità del Neolitico è ricostruita da Marion Benz che, su base antropologica e neurobiologica, analizza la medialità dei simboli: l'esistenza di "anthropological patterns" costanti rende infatti possibile l'interpretazione dei simboli e delle reazioni da essi provocati al di là delle specifiche differenze culturali. Benz nota in concomitanza con la sedentarizzazione, un incremento nell'uso di simboli, volto a creare un'identità comune al di sopra del gruppo ristretto, primo passo verso l'introduzione di codici morali e sociali, che porteranno allo sviluppo del discorso religioso. Bo Dahl Hermansen concorda con Marion Benz nell'individuare un crescente uso di linguaggio simbolico figurato durante il Neolitico. Analizzando i resti umani e architettonici di sepolture nel sito di Shkārat Msaied, Hermansen mostra 
come gli abitanti neolitici del sito condividessero un sistema di norme e credenze 'istituzionalizzate', in un quadro che rende possibile la qualifica della loro vita spirituale come religione: in particolare l'architettura funeraria, le testimonianze relative al consumo di pasti rituali e la manipolazione/smembramento di alcune parti del corpo indicano la costruzione di una memoria culturale in cui i morti costituivano un tramite tra il mondo dei vivi e l'aldilà. Tre contributi (Flemming Kaul, Mads Kähler Holst e Klaus Randsborg) affrontano il tema della mitologia e delle pratiche rituali nell'Età del Bronzo scandinava, con particolare attenzione al culto del Sole.

I contributi della terza sezione ("Text"), affrontano il tema della materialità della religione in epoca storica e in culture in cui la scrittura è il principale medium per l'espressione e la conservazione del pensiero religioso. Izaak J. De Hulster, partendo dall'analisi dell'Antico Testamento, sottolinea a più riprese, come, poiché gli agenti della religione sono umani, una religione che non abbia alcuna forma di fisicità (p. 288: "location and embodiment") è semplicemente impossibile, anche se certamente alcune forme di materialità lasciano tracce più evidenti di altre. Le immagini ("pictoriality") costituiscono secondo l'autore un ponte tra la materialità dell'oggetto e l'immaterialità del pensiero scritto (ma nel momento in cui il pensiero diviene testo, non assume anch'esso forma materiale?). La pittorialità può dunque essere considerata come una forma ibrida tra espressione materiale e immateriale, tra materialità e testualità (l'immagine, tuttavia, presupponendo un supporto, non è anch'essa un artefatto?). A seguito dell'“aniconic turn", dell'Antico Testamento, messo dall'autore in relazione diretta con gli sviluppi monoteistici, vengono esplorati nuovi modi di relazionarsi alla materialità. Tuttavia, sebbene l'aniconismo esprima certamente una forma particolare di materialità, va notato che la presenza o assenza di immagini non qualifica una religione come più o meno materiale. Il tema della materialità del testo è affrontato da Trine Bjørnung Hasselbalch che analizza i manoscritti del Mar Morto (e in particolare l'apocrifo della Genesi), come artefatti materiali e, nello specifico, come palinsesti, mostrando come questo cambio di prospettiva renda possibile una nuova percezione del testo e del suo ruolo come scrittura religiosa all'interno della comunità locale. Lars Östman infine, in un contributo che è l'unico dedicato al mondo contemporaneo e a una pratica onoraria, più che religiosa, tenta, tramite un ardito parallelo con la religione romana arcaica, di sottolineare il carattere rituale ("profane rituality") delle Stolpersteine che dal I992 commemorano sulle strade d'Europa le vittime dell'Olocausto. L'autore si chiede attraverso quale paradigma religioso possa essere compreso questo fenomeno di memoria culturale e storica, che si autodefinisce "ein Kunstprojekt für Europa”. Ma a parte l'uso della definizione di ritualità profana per le Stolpersteine (una definizione che in realtà si adatta a qualsiasi pratica commemorativa), l'autore non specifica quale sia il carattere specificatamente sacro dell'opera, che viene dato per scontato.

Il volume è ricco di materiale fotografico di buona qualità ed è completato da un breve indice analitico. In generale, nonostante alcune debolezze (una definizione del concetto di materialità e una sintesi del dibattito critico sugli aspetti materiali della scrittura avrebbero evitato molta confusione), non si può che apprezzare una pubblicazione che si propone coraggiosamente di riaprire la discussione su un tema complesso e scarsamente trattato.

Irene BERTI

Pädagogische Hochschule Heidelberg Institut für Gesellschaftswissenschaften (IGW), Abteilung Geschichte (GES) ireneberti1@yahoo.it 
Guillaume BudÉ, De asse et partibus eius. L'As et ses fractions. Livres I-III, éd. et trad. L.-A. SANCHI, Genève, Droz, 20I8, CXLVIII+592 p., 79 €/ISBN 9782600058773 .

Avec les Commentaires de la langue grecque de Guillaume Budé (voir la recension de Françoise Frazier, Anabases, 8, 2008, p. 277-279), Luigi-Alberto Sanchi, actuellement directeur de recherches au CNRS, avait déjà fourni en 2006 un outil de travail remarquable aux chercheurs de toutes disciplines s'intéressant à l'humanisme du $\mathrm{xvI}^{\mathrm{e}}$ siècle en France. Poursuivant son travail d'édition et de traduction des œuvres de l'humaniste français et complétant ainsi les éditions de textes et monographies dues à MarieMadeleine de la Garanderie, il livre aujourd'hui, en une édition abondamment documentée et accompagnée d'une traduction, les trois premiers des cinq livres du De asse et partibus eius, plus couramment intitulé De asse.

Si le titre annonce un traité de numismatique, la perspective de Budé est bien plus large et se caractérise par son " ouverture disciplinaire: un ouvrage mêlant histoire, économie, lettres et philologie» (p. VII). Ainsi les longs excursus sur les monnaies et mesures de l'Antiquité voisinent avec des pages consacrées au luxe des Anciens (un passage du livre II traite par exemple des finances de Jules César) ou avec l'étude de questions philologiques, entre autres sur le texte d'un extrait du livre XXXIII de Pline l'Ancien dont Budé discute les difficultés d'éatablissement et d'interprétation au début du livre III de son ouvrage. On rencontre également dans la seconde partie du premier livre de longs développements sur la dignité et la valeur de la contribution française aux sciences et aux lettres, appuyés sur la « revendication d'un humanisme français non moins autorisé que l'italien » (p. XI).

Ce volume s'ouvre sur une vaste introduction, complétée de cinq annexes, qui jette tout d'abord un éclairage fort utile sur la place du De asse dans l'œuvre de l'humaniste français. Vient ensuite une longue mise au point sur la rédaction et les différentes éditions imprimées du texte et sur les remaniements opérés par l'auteur au fil des années (p. XVII-XXIX). Un travail de recherche extrêmement précis et riche a été mené sur les sources utilisées par Budé (p. XXIX-XLV). Enfin, le lecteur trouvera un intéressant développement sur la fortune de l'ouvrage (p. XLV-LII).

Sont particulièrement appréciables la clarté et la précision des annexes qui offrent au lecteur moderne de précieux guides pour s'y retrouver dans ce texte touffu, à l'érudition dense et ardue : pour n'en citer que deux, la première annexe propose un bref panorama du contenu des cinq livres, suivi d'un plan détaillé qui met en relief l'organisation de l'ouvrage et la construction de son argumentation (p. LIXLXV); la quatrième annexe, fruit d'un minutieux travail de recherche, offre au lecteur une liste des « auteurs cités, sources de Budé et éditions modernes ", classée par ordre alphabétique d'auteurs (p. XCICXLVI). Elle met en lumière la variété et la qualité de l'érudition de l'humaniste français.

Pour l'édition critique du texte, L.-A. Sanchi part de la dernière version du texte revue par Budé lui-même, à savoir l'édition de I54I, parue à Paris, chez Michel de Vascosan, RobertEstienne etJean deRoigny. L'apparat critique fait apparaître les ajouts et remaniements des éditions successives, de la première imprimée en i5ı5 à l'édition posthume de i54r. Pour le balisage du texte, l'éditeur et traducteur a choisi la pagination in- $8^{\circ}$ de l'édition de Lyon, i55o-i55 ı qui permet un maillage précis du texte original. La troisième annexe à l'introduction offre un tableau de correspondance des pages entre les différentes éditions qui permet de retrouver aisément un passage du texte dans n'importe laquelle des éditions anciennes. Le texte original, latin émaillé de 
citations grecques, est accompagné d'une belle traduction française qui se distingue autant par son élégance que sa précision. Le latin de Budé est ardu et le texte fort long. On ne peut donc que rendre hommage à ce patient travail de traduction qui met ce texte d'importance à la disposition d'un grand nombre de lecteurs, quelle que soit leur maîtrise de la langue latine.

Cette très belle édition de ce chef d'œuvre de Guillaume Budé est un outil de référence qui profitera non seulement aux chercheurs de toutes les disciplines des lettres et sciences humaines, mais aussi aux curieux qui s'intéressent à la Renaissance française et à l'émergence de l'intérêt pour les sciences de l'Antiquité.

Laure Hermand-Schebat Université de Lyon-Jean Moulin-Lyon 3 laure.hermand@univ-lyon3.fr

\section{Maurizio Campanelli et Frances Muecke (éds.), The Invention of Rome: Biondo Flavio's Roma Triumphans and Its Worlds, Genève, Librairie Droz, 20I7, 292 p., $48 \mathrm{CHF} / \mathrm{ISBN} 9782600047890$.}

Cet ouvrage est la publication des actes d'une rencontre scientifique ayant eu lieu à la British School de Rome en 20r4. Son but est de réévaluer la nature, le contenu, les idées principales et la réception de la Roma triumphans de Flavio Biondo (I392-I463), le dernier ouvrage majeur de l'humaniste italien, écrit en I 459 , et considéré comme une œuvre essentielle de l'humanisme et comme le fondement de l'antiquarisme. La brève introduction (p. 9-15) rappelle l'importance de l'œuvre, tout en soulignant sa postérité actuelle limitée. L'ouvrage souhaite à la fois comprendre la réception de la Rome antique au $\mathrm{xv}^{\mathrm{e}} \mathrm{s}$., tout en améliorant la connaissance du contexte d'écriture.

Le livre est divisé en trois parties : "Context, Genre, and Purpose» (p. 19-73), “Mores et instituta» (p. 77-195) et “ Reception » (p. 199-273). La première partie s'intéresse plus spécifiquement à la contextualisation de la rédaction de l'ouvrage, en le replaçant dans son contexte culturel, historique, littéraire et politicoreligieux. L'article d'A. Raffarin a déjà été publié et a subi de légères modifications (“La célébration des triomphes de Rome par Flavio Biondo dans la Roma instaurata et la Roma triumphans »). Les références à l'Antiquité doivent servir le projet de réinstauration de la primauté romaine et de restitution de l'“aura antique» (p.20). Biondo a en effet été secrétaire à la curie pontificale (1435-1460); de fait il montre le triomphe d'une Rome chrétienne restaurée par l'autorité papale et s'inscrit dans l'ancienne tradition des éloges des cités. Puis F. Muecke s'interroge sur la complexité de l'ouvrage et sa catégorisation, car il est difficile de déterminer s'il s'agit d'une laus Romae, une histoire ou une œuvre antiquaire (“The Genre(s) and the Making of Roma triumphans »). Plus qu'une compilation, c'est une immersion dans la culture romaine à l'époque humaniste. La conclusion de l'ouvrage établit un lien entre la République romaine et la Rome chrétienne. A. Mazzocco propose de revenir sur ce passage très étudié et sur la comparaison en la replaçant dans le contexte d'écriture, y compris géopolitique avec la menace turque (“The Rapport between the Respublica Romana and the Respublica Christiana in Biondo Flavio's Roma triumphans »).

La deuxième partie “Mores et instituta» est plus conséquente avec six contributions qui explorent les principales thématiques de la Roma triumphans: la religion, la politique, l'armée, l'espace public, les monuments de la Ville éternelle... La première contribution, “Gentiles nostri: Roman Religion and Roman Identity in Biondo Flavio's Roma triumphans ", a déjà été publiée. Les deux premiers livres consacrés à la religion romaine ont peu suscité l'intérêt. F. Muecke en propose une 
relecture passant par les sources utilisées par Biondo, notamment Eusèbe de Césarée, avant de s'intéresser aux similitudes entre cultes païens et chrétiens grâce à la survivance de certains aspects ou pratiques. Ensuite J. Hankins s'intéresse à la teneur de la res publica pour Biondo, en la replaçant dans la conception de la constitution mixte romaine (“Biondo Flavio on the Roman Republic »). Selon Biondo, la puissance romaine ne relevait pas de son système politique ou de la liberté, mais du respect d'un certain nombre de vertus. La littérature antique est au cœur de l'article de G. Marcellino ( Un excursus umanistico sulle letterature dell'antichità : Biondo Flavio e i classici (Roma triumphans IV, pp. 96-ıоo)». Biondo a utilisé de nombreuses sources dans un contexte de redécouverte humaniste d'une Rome antique valorisée. Puis les aspects militaires sont abordés par I. G. Mastrorosa ( Roman Military Discipline in Biondo Flavio's Roma triumphans: Punishments and Rewards »). Elle montre le cheminement intellectuel de l'auteur utilisant les sources antiques pour donner des exemples précis. Pour Biondo la discipline est un point central du système militaire romain et a grandement contribué aux victoires. M. Campanelli analyse ensuite une controverse opposant Biondo et Francesco Barbaro sur fond de vestiges antiques à Rome («Il libro IX della Roma triumphans: una querelle umanistica degli antichi e dei moderni »). Cela révèle l'écart entre les attitudes humanistes et médiévales face à cette monumentalité antique. Selon Biondo, l'architecture antique représente une forme de perfection quasiment inatteignable à son époque. Ces réflexions doivent être replacées dans celles du Quattrocento, notamment avec l'œuvre de Léon Battista Alberti (De re aedificatoria). L'architecture est aussi au cœur de l'article suivant. P. Fane-Saunders montre que Biondo reprend trois catégories antiques : la domus, le rogus (le bûcher funéraire des empereurs permettant de parler des funérailles impé- riales) et la villa (« Pyres, Villas, and Mansions: Architectural Fragments in Biondo Flavio's Roma triumphans »).

Enfin la dernière partie s'intéresse à la réception de la Roma triumphans grâce à quatre contributions. D'abord les manuscrits conservés sont étudiés par M. A. Pincelli (“"Librariis certatim transcribere contendentibus" : la tradizione manoscritta e la prima ricezione della Roma triumphans di Biondo Flavio »). Quelques pages sont même reproduites (p. 209-2I2). P. Gwynne revient ensuite sur la volonté de Biondo d'appeler à une nouvelle croisade et la comparaison avec un "triomphe romain» en I472 et la figure de Jules II présenté comme un triomphateur («Triumphs and Triumphators in the Wake of the Roma triumphans »). La postérité est également étudiée à travers l'exemple d'A. Fulvio (Antiquitates urbis, I527) par A. Raffarin (“ Fulvio lecteur de Biondo : questions religieuses dans la Roma triumphans et les Antiquitates urbis »). Même si de prime abord l'ouvrage est consacré à la topographie de l'Vrbs, il a pu s'inspirer de Biondo dans plusieurs domaines comme la religion. L'article insiste aussi sur les sources antiques mobilisées. Enfin W. Stenhouse s'intéresse à la

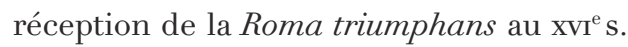
(“Flavio Biondo and Later Renaissance Antiquarianism »). Même si la dernière édition latine a été imprimée en 5559 , les antiquaires continuèrent à la lire et à la citer.

Pour conclure, l'approche diachronique incluant l'analyse de l'antiquité romaine, le contexte d'écriture de la Roma triumphans et sa postérité permet d'offrir aux lecteurs, à travers des études de cas précises, un panorama important du dernier ouvrage de Flavio Biondo.

Cyrielle LANDrEA

Université Bretagne Sud cyrielle.landrea@univ-ubs.fr 
Jean-Noël Castorio, Rome réinventée. L'Antiquité dans l'imaginaire occidental de Titien à Fellini, Paris, Vendémiaire, 20I9, 439 p., 24€/ISBN 9782363583284.

Maître de conférences en histoire antique à l'université du Havre, remarqué pour ses biographies de Caligula et Messaline au cours desquelles il s'intéressait déjà à la réception de la mémoire de ces deux personnages, Jean-Noël Castorio nous propose un ouvrage qui s'inscrit pleinement dans les thématiques chères à la revue Anabases. De l'époque du Titien à la filmographie de Federico Fellini, l'auteur propose onze contributions qui s'organisent à la fois avec une présentation du dossier antique et une analyse de la réception dont le sujet a bénéficié. Du viol de Lucrèce par Sextus Tarquin à la fin du monde romain, en passant par la révolte des mercenaires de Carthage, Spartacus, l'assassinat de César, les proscriptions des guerres civiles, les larmes d'Hadrien, Héliogabale et la Rome antique de Fellini, Jean-Noël Castorio nous emmène dans un voyage culturel extrêmement divers, au contact d'œuvres majeures que sont Histoire du déclin et de la chute de l'Empire romain de Gibbon, le tableau Les Romains de la décadence de Thomas Couture, Salammbô de Flaubert, les tragédies de Shakespeare Le jeune roi et Titus Andronicus, Les Mémoires d'Hadrien de Marguerite Yourcenar, le film de Fellini Satyricon. Le livre est conçu comme un ensemble d'études indépendantes qui peuvent être lues au gré des envies du lecteur et l'auteur du présent compterendu a abordé le livre par «La Rome antique de Fellini » et « La fin d'un monde ». Dans chaque contribution, Jean-Noël Castorio présente l'état des connaissances afin d'éclairer le sujet antique. Ainsi, pour le Satyricon de Fellini projeté sur les écrans en I969, si l'auteur ne tranche pas sur la période néronienne, flavienne et du début $\mathrm{du} \mathrm{II}^{\mathrm{e}}$ siècle de la rédaction, il présente le contenu des extraits conservés de l'œuvre et décortique la manière dont Fellini en a tiré son film avec pour principe que «le monde antique n'a jamais existé, nous l'avons rêvé », renouant avec des pratiques du néo-réalisme italien en prenant ses acteurs parmi des "gens de la rue» tel Mario Romagnoli, patron d'une trattoria romaine et qui incarne Trimalcion ou des acteurs sans passé notable à l'image de l'Américain Hiram Keller dont le « principal fait d'armes était d'avoir participé au chœur des hippies dans la comédie musicale Hair » (une exception notable cependant avec la participation d'Alain Cuny). De même dans la contribution intitulée “Les larmes d'Hadrien » et consacrée à la relation amoureuse entre l'empereur Hadrien et Antinoüs, Jean-Noël Castorio analyse la genèse des Mémoires d'Hadrien, ouvrage que Marguerite Yourcenar publie en ig5r et présente les diverses sources antiques qui nous permettent de connaître cette idylle qui fit d'Antinoüs, à la suite de sa noyade accidentelle dans le Nil, un dieu. Mais il présente également les prises de position des sources antiques du sévère Dion Cassius aux auteurs chrétiens tels Symmaque, Tertullien, Clément d'Alexandrie, Aurelius Victor. «Si certains apologistes chrétiens regardaient donc Antinoüs comme une victime de la lubricité d'Hadrien, tandis que d'autres le jugeaient coupable d'en avoir été le complice actif, ils étaient en revanche unanimes à considérer sa divinisation comme l'un des meilleurs témoignages de la perversion du paganisme» (p. 272). Dernier exemple, "Le règne scandaleux du Roi-Soleil » c'est-à-dire la figure de l'empereur Héliogabale (2I8-222). Ce règne jugé hautement sulfureux a inspiré le tableau de Lawrence Alma-Tadema, présenté à la Royal Academy of Arts de Londres en i888. Jean-Noël Castorio offre une analyse précise du contexte et de l'œuvre elle-même, puis présente l'image d'Héliogabale dans les écrits des historiens antiques qui "apportent une réponse unanime et sans nuance : il fut un monstre 
comme les Romains, pourtant prompts à remettre leur destin entre les mains de tyrans sanguinaires, n'en avaient jamais connu » (p. 337).

Riche en références littéraires et artistiques, le livre de Jean-Noël Castorio participe pleinement à l'approfondissement de la question de la réception et de la construction des perceptions de l'Antiquité qui a trouvé désormais sa place dans le paysage historiographique de la période ancienne.

\section{Philippe Foro \\ Université de Toulouse - Jean Jaurès philippe.foro@wanadoo.fr}

\begin{abstract}
Ettore Cinnella, Lo zar e il latino. Gli studi classici in Russia tra Otto e Novecento, Pise et Florence, Della Porta Editori, 2018, 26o p., I7,70 €/ ISBN 9788896209318.
\end{abstract}

Studioso di storia russa (in particolare delle rivoluzioni del igo5 e del I9I7) e appassionato cultore di antichistica, Ettore Cinnella propone ne Lo zar e il latino una densa sintesi dell'evoluzione degli studi classici in Russia tra la seconda metà del XIX e i primi decenni del XX secolo. Il volume si situa nell'alveo di una ricca tradizione di ricerche in cui la vicenda della ricezione del mondo classico in Russia si connette con l'indagine sulle riforme scolastiche liceali di volta in volta destinate a promuovere o a limitare l'insegnamento delle lingue greca e latina: ricca tradizione che presenta al suo interno una grande varietà di espressioni storico-storiografiche, come ad es. i lavori di Frolov e di Wes, del cui Classics in Russia (1992), incentrato sul periodo I700-I855, Lo zar e il latino costituisce - almeno sul piano cronologico - una continuazione suigeneris.

L'intreccio tematico-prosopografico proprio di questo filone di ricerche presenta, nel volume di Cinnella, aspetti di ragguardevole originalità. In primo luogo all'esasperata erudizione e al paratattico affastellamento di dati viene preferita una visione impressionistica dei temi e degli autori presi in esame. Secondariamente gli approfondimenti prosopografici vengono da Cinnella rivolti a studiosi - esponenti di discipline, di metodologie e di indirizzi differenti - non sempre sufficientemente conosciuti o valorizzati dagli antichisti "occidentali". Per limitarmi ad alcuni esempi tra i numerosissimi trattati, ricorderò V.I. Modestov, filologo-storico-archeologo formatosi alla scuola di Fr. Ritschl e di O. Jahn, studioso di Tacito e di preistoria romano-italica abbastanza apprezzato da Rostovtzeff (Lo zar, cap. 4) e da S. Reinach; E.D. Grimm, "antimommseniano" autore di un'opera-incentrata sulla natura giuridicoistituzionale del principato - recensita e pervicacemente contestata da Rostovtzeff (cap. 9); M.M. Chvostov, filologo e storicoeconomista, che alla svolta del secolo studiò il commercio dell'Egitto ellenistico e, nel dibattito allora infuocato tra "primitivisti" e "modernisti" (cap. 8), assunse una posizione moderatamente meyeriana, simile a quella cui sarebbe pervenuto l'ultimo Rostovtzeff. Sostanzialmente bücheriano, oltre che "fusteliano", fu invece I.M. Grevs, alla cui figura di studioso Cinnella riserva ben 3o pagine del cap. 8, giustamente enfatizzando l'importanza della sua (incompiuta) opera sulla struttura della proprietà fondiaria a Roma. Come ricorda Cinnella, spettò al filologo russo-polacco Zielinski, di fede "meyeriana" e maestro di Rostovtzeff, render conto in un'unica recensione del ponderoso volume di Grevs (I899) e della coeva Geschichte der Staatspacht del ventinovenne Rostovtzeff, che l'anno successivo avrebbe pubblicato il saggio Capitalismo ed economia nazionale nel mondo antico, di spiriti decisamente meyeriani. Zielinski ovviamente svalutava le tesi di Grevs a beneficio di quelle del discepolo, con il quale, tra l'altro, collaborava nello stesso I899 alla traduzione russa del Grundriss di B. Niese. In ogni caso, 
le divergenze di opinioni sull'economia antica non impedirono a Rostovtzeff e a Grevs di intrattenere un rapporto di stima e di rispetto, forse anche propiziato dal comune amico Vjačeslav I. Ivanov, il cui carteggio con Grevs fu edito nel 2006 nello splendido volume Storia e poesia, curato da G. Bongard-Levin, N.V. Kotrelev ed E. Liapustina.

Da quanto si è detto emerge dunque con chiarezza che il terzo e più rilevante motivo di originalità nel volume di Cinnella consiste nella presenza di un filo conduttore, o meglio di un "nucleo di riferimento" rappresentato dalla straordinaria figura e dall'opera di M. Rostovtzeff, in cui con esattezza si ravvisa, in certo modo "teleologicamente", una sorta di bacino collettore delle problematiche, dei dibattiti, delle polemiche che caratterizzarono l'antichistica russa nel xIx secolo (almeno dall'epoca di S.S. Uvarov) e nel primo ventennio del xx. La presenza di Rostovtzeff non solo è dominante in tutta la seconda parte del libro, ma è evidente anche nella prima parte, che verte sulla tormentata vicenda delle cause e degli effetti dell'insegnamento del latino e del greco nei licei e sul tortuoso itinerario del classicismo russo (capp. 2 e 3). Non è ad es. casuale che il cap. 3 del volume - in cui si delinea l'evolversi del classicismo in rapporto agli ordinamenti delle scuole superiori nella Russia dell'ultimo trentennio del xix secolo, dalla riforma del I87i alla stretta reazionaria degli anni Ottanta alle aperture "liberali" che finalmente limitarono ai licei classici l'apprendimento del greco - si chiuda con una testimonianza di Rostovtzeff sulla coraggiosa opposizione di V.I. Modestov all'ultraconservatrice e ormai anacronistica organizzazione degli studi liceali nella Russia di I.I. Tolstoj e di I. D. Deljanov. Allo stesso modo non è casuale che il volume di Cinnella, chiuso da un Epilogo interamente dedicato a Rostovtzeff, si apra con un capitolo costruito sull'Autonecrologio di S.A. Žebelëv, lo studioso che per carattere, interessi scientifici e atteggiamenti politici e accademici rappresentò il contraltare, il "gemello diverso" di Rostovtzeff.

Nel mezzo secolo che precedette il I9I7 l'antichistica pietroburghese costituì l'esito qualitativamente più significativo dell'affermazione-forzosamente imposta da Pietro il Grande e un secolo dopo riaffermata nelle riforme di S.S. Uvarov nel I8II - di un modello sostanzialmente "occidentalista" di studio delle lingue e della civiltà classiche (che in Uvarov peraltro si accompagnava a un vivissimo interesse per l'Oriente), esemplato prevalentemente su precedenti tedeschi; un modello che ovviamente fu avversato in modi e misure diverse da molte componenti dell'intelligencija (a cominciare da "orientalisti" e da "slavofili") che consideravano trascurata o depressa la prismatica varietà della cultura e della spiritualità russe. Diffuso da maestri germanici (come Graefe, Nauck, L. Müller, Ritschl) o di formazione germanica (come Zielinski) nelle Università russe e in centri come Dorpat e Leipzig, il "metodo tedesco" - come ricorda Cinnella nei capitoli centrali del suo libro - veniva assorbito dagli antichisti pietroburghesi (da M.S. Kutorga a F.F. Sokoloff, da V.V. Latyšev e V.K. Jernstedt a G.F. Zereteli e M.I. Rostovtzeff) anche nel corso di lunghi viaggi di istruzione all'estero, e specialmente in Germania, in Grecia e in Italia. Il rigore metodico della scuola storico-filologica di Pietroburgo da un lato tendeva a creare un solco tra lo studio scientifico dell'antico e lo studio della "storia generale" di tipo "granovskijano" (e in parte anche quello del "filone politicosociale" cui Cinnella accenna a proposito di V.P. Buzeskul), dall'altro, per merito di F.F. Sokoloff, N.P. Kondakov e discepoli (i presunti faktopoklonniki = "adoratori dei fatti”), consentiva di pervenire, tramite minuziose indagini, a solide e dettagliate descrizioni di aspetti e problemi del mondo classico. Associando i principi della Altertumswissenschaft a una moderata dose di sano positivismo (entrambi indigesti a chi, nel nome di una fantomatica 
"tradizione russa", agitava il mito polemico dei faktopoklonniki), la fucina accademica pietroburghese perseguiva un ideale di ricostruzione dell'antico necessariamente fondata su preliminari indagini "a tappeto" sul piano documentario e bibliografico e sulla conoscenza diretta, de visu, di tutte le possibili fonti di informazione (da quelle letterarie a quelle archeologiche ed epigrafiche a quelle iconografiche): di qui la fioritura o la rifioritura, su basi scientifiche "occidentali", di discipline come l'epigrafia greca (e latina), la paleografia, la papirologia (si pensi a Zereteli, allievo di Jernstedt) e soprattutto l'archeologia, che con la filologia classica Rostovtzeff considerava il fondamento del sapere storico. Alle ricerche di storia antica (soprattutto greca), si accompagnarono quelle interdisciplinari su Bisanzio, sulla (allora) Russia Meridionale e sulle coste del Mar Nero, e cioè, come giustamente indica Cinnella, su un enorme quadrante geografico e su un arco cronologico più che millenario la cui antica e complessa storia veniva considerata parte integrante delle "radici russe" (Lo zar, cap. 7). Tra gli artefici di questi studi sono da ricordarsi almeno il filologo classico e bizantinista V.K. Jernstedt - maestro di Rostovtzeff e di Zebelev - e il suo compagno di studi e bizantinista Latyšev, che si dedicò anche all'epigrafia greca del Mar Nero; l'archeologo B.V. Farmakovskij, che scavò a Olbia Pontica, e soprattutto N.P. Kondakov, archeologo e studioso di arte classica e soprattutto di iconografia bizantina, che trasmise a Rostovtzeff e a J.I. Smirnov la capacità di vedere "con occhio d'aquila" il monumento o l'oggetto antico e di indagarne tutti i possibili aspetti e significati, da quello artistico a quello economico-sociale a quello spirituale o religioso. Mai Rostovtzeff venne meno al principio kondakoviano della storia narrata anche per immagini, come mostrano gli apparati iconografici non solo delle opere su Pompei, sulla Russia Meridionale e su Dura Europos, ma anche delle sue notissime sintesi sull'impero romano e sul mondo ellenistico.

Intorno alla sezione finale de Lo zar e il latino, interamente dedicata a Rostovtzeff, non mi soffermo ulteriormente anche perché molti dei temi cui Cinnella accenna sono ripresi (Lo zar, p. I33) dalle discussioni presenti nell'enorme produzione di studi rostovtzeviani dell'ultimo quarto di secolo: molto è stato scritto, ad es., sulla formazione culturale e scientifica e sul problema delle "radici russe" del grande storico (che Cinnella tende a sottovalutare), sulle sue probabili simpatie "eurasiane" (evidenti nella Prefazione a $A$ History of Russia di Vernadskij del I929), sulla sua conversione politica-ideologica - determinata dalle tragedie della guerra e della rivoluzione - dal cosmopolitismo della giovinezza al nazionalismo della maturità, dal "cadettismo" liberal-democraticocostituzionalista a posizioni fortemente conservatrici e addirittura reazionarie (si pensi all'ammirazione per Mussolini).

Intendo qui focalizzare l'attenzione sulle pagine che Cinnella destina a $L a$ nascita dell'impero romano, l'operetta sulle guerre civili e sul principato augusteo che Rostovtzeff licenziò il 25 maggio ı9ı, un mese prima della partenza per l'esilio (3o giugno).

Come sui temi sopra accennati, pure su questo testo sono anch'io più volte intervenuto (in contributi editi nel I999, nel 200I, nel 2003, nel 2012 e nel 2018 e ora ristampati in P.G. Michelotto, Studi su M.I. Rostovtzeff, "Bruno Mondadori”, Milano 2org) e constato dunque con soddisfazione che Cinnella è pervenuto, nell'interpretazione de La nascita, a conclusioni che collimano quasi perfettamente con le mie. Forse l'unico motivo di profondo dissenso è rappresentato dal valore da attribuirsi all'opuscolo, che Cinnella (p. 222) colloca - a mio avviso a torto - "tra le cose migliori di Rostovtzeff".

Essenzialmente esatte in Cinnella (anche se prive di qualche doverosa nuance) sono 
invece la consapevolezza dell'impossibile "sovrapponibilità" della rivoluzione russa alla "rivoluzione romana" (e viceversa) e, di conseguenza, l'opinione secondo cui la rievocazione delle guerre civili del i a.C. e l'interpretazione del principato augusteo, per quanto ovviamente contengano inevitabili allusioni al "clima" del presente rivoluzionario, non siano da considerarsi dettate e "marchiate" dalla rivoluzione in corso, ma da vedersi come uno svolgimento coerente del pensiero di Rostovtzeff, che su questi argomenti tornò almeno sei volte tra il ı899 e il ı9ı8 (per i dettagli rimando agli Studi su M.I. Rostovtzeff sopra menzionati). Da ciò scaturisce l'ovvio corollario che, ne La nascita dell'impero romano, nulla autorizza a vedere in trasparenza, in Augusto e in altri protagonisti della "rivoluzione romana", personaggi o momenti politici della rivoluzione russa. Come ho più volte asserito, ribadisco, con Cinnella, che l'Augusto di Rostovtzeff non fu né un Kerenskij né un Kornilov.

Pier Giuseppe Michelotto Università degli Studi di Milano piergiuseppe.michelotto@unimi.it

Bianca De Divitiss, Fulvio Lenzo et
Lorenzo Miletti (éds.), Ambrogio Leone’s
De Nola, Venice I5I4. Humanism and
Antiquarian Culture in Renaissance
Southern Italy, Leyde et Boston, Brill,
20I8, 256 p., $94 €$ / ISBN 9789004375789.

Dans la lignée de Flavio Biondo et de son Italia illustrata de I474, les humanistes consacrent des écrits à vocation archéologique tout au long de la Renaissance, témoignant de l'émergence d'une culture antiquaire. C'est le cas d'Ambrogio Leone (Nola I458 - Venise i525) qui a dédié un traité à sa ville natale, le $D e$ Nola publié à Venise en i5ı4. Ce traité n'a fait l'objet d'aucune étude complète, ce à quoi remédie cet ouvrage collectif, fruit des recherches d'un groupe de travail interdisciplinaire et de deux séminaires organisés à Nola et à Venise en 2012.

En effet, l'ouvrage propose pour la première fois une analyse exhaustive du livre de Leone qui, s'il est peu connu, fut considéré au $\mathrm{xvI}^{\mathrm{e}}$ siècle comme an important advance in European humanistic and antiquarian debates : il le replace ainsi dans son contexte historique, culturel et éditorial, tout en en soulignant l'originalité générique et littéraire. La méthode choisie par les auteurs est tout aussi pertinente: le choix de l'interdisciplinarité permet de souligner l'importance d'une œuvre considérée comme mineure. Aussi le lecteur la découvre-t-il selon des entrées transversales: la bibliographie matérielle du livre, ses sources et modèles, ses références culturelles et artistiques, le rôle des illustrations, le contexte historique, social et anthropologique de sa réalisation. Ce choix permet de rendre compte de l'approche encyclopédique et humaniste du livre en huit études explicitant la complexité et l'originalité de l'œuvre dans toutes ses dimensions, tout en montrant en quoi elle témoigne de la circulation des idées en Europe et de l'importance des relations entre le nord et le sud de l'Italie.

L'introduction permet de mieux cerner l'auteur et son traité: Leone, médecin évoluant dans le milieu humaniste napolitain, et parti au début du xvi ${ }^{\mathrm{e}}$ siècle à Venise pour une raison inconnue, fréquente Alde Manuce et son Académie. Il écrit des ouvrages sur la médecine et la philosophie aristotélicienne, à l'exception de sa première publication, le De Nola. Celui-ci est divisé en trois livres, de quinze chapitres environ chacun : une description de la ville antique, celle de la ville contemporaine et, un livre sur les habitants, coutumes et traditions, le tout accompagné de quatre illustrations, en faisant à la fois un livre scientifique et un objet d'art. 
L'étude du De Nola s'articule en huit chapitres qui offrent un parcours interdisciplinaire. Le premier porte sur l'identité générique complexe du livre, ses sources antiques et sa vocation encomiastique et morale. Antiquité et période contemporaine sont vues dans un continuum caractéristique de l'approche humaniste du traité, ce qui amène ensuite à préciser la méthode suivie par l'antiquaire. Celui-ci parvient à reconstruire la gloire passée de l'antique Nola en s'appuyant sur les sources écrites, les traces archéologiques et les édifices contemporains, preuve de la magnificence commune de la Nola vetus comme de la Nola praesens. Fulvio Lenzo revient quant à lui sur la fonction des quatre gravures de Mocetto, nouveauté éditoriale pour ce type d'ouvrages, qui constituent une mise en image de la méthode et de la pensée de l'antiquaire. D'ailleurs, l'examen des descriptions architecturales de Nola depuis l'Arx aux domus, en passant par la Regia et la cathédrale est un exemple parlant de cette méthode: archéologie et chorographie mises en parallèle avec la topographie sociale servent ici un discours de célébration de la noblesse locale, la splendeur architecturale passée et présente étant le reflet de celle de ces nobles habitants. Après une ouverture sur Leone et les arts de son temps, les deux derniers chapitres portent sur le livre III du De Nola. Ainsi, Giuliana Vitale démontre notamment comment le traité s'appuie sur un propos scientifique éclairant l'histoire politique et socio-économique pour fixer la mémoire de la ville, le document devenant monument à la gloire de la dignitas des habitants. Cette visée est renforcée par l'analyse des rites et fêtes de Nola qui permettent à nouveau de créer un parallèle entre Antiquité et présent au service d'un discours patriotique. Une notice bibliographique de l'édition de ı5ı4 parachève l'ensemble qui est complété par de nombreuses annexes comportant des extraits du De Nola, des illustrations, une bibliographie et un index.
Les auteurs proposent donc un ouvrage clair et complet, permettant de découvrir un traité qui est ainsi réévalué à l'aune de l'importance qu'il a eue, véritable jalon dans la culture humaniste et antiquaire de la Renaissance et prouvent en même temps la fécondité de ce type d'approche interdisciplinaire pour les spécialistes de la période, dans la lignée des réflexions initiées lors du colloque L'étude la Renaissance nunc et cras en 200I.

\section{Clarisse Évrard \\ Université de Lille - École du Louvre clarisse.evrard@wanadoo.fr}

\author{
Michael A. Flower (éd.), The Cambridge \\ Companion to Xenophon, Cambridge, \\ University Press, 20I7, xx+520 p., £87,99 \\ (hardback) ou £28,99 (paperback) / \\ ISBN 978IIo7050o68 (hardback) ou \\ 9781ıо7652ı56 (paperback).
}

Vingt-deux auteurs publient dans ce volume, à la fois dense et cohérent, vingtdeux articles (dont deux sous la plume de Tim Rood) et un épilogue qui - comme il se doit dans un companion - donnent une très complète introduction à l'œuvre de Xénophon (ci-après X.). L'introduction par Michael A. Flower (qui signe aussi une contribution) met l'accent sur la réception de X. et l'histoire de ses lectures modernes. Pourquoi X. intéresse-t-il autant, bien au-delà des milieux spécialisés, et ce depuis longtemps? À quels défis sont confrontés ses interprètes et quelles perspectives nouvelles se dessinent pour son étude? L'ouvrage se donne donc pour ambition de s'adresser à la fois aux spécialistes, aux étudiants et au grand public. Il est en outre conçu pour traiter l'œuvre de X. dans son ensemble, même si les œuvres particulières sont présentées et traitées également dans leur singularité d'objet et de style (section II). 
Les contributions sont regroupées en cinq sections thématiques de longueur relativement comparable, à l'exception de la dernière, consacrée à l'influence et à la réception de Xénophon, relativement plus courte. On passe ainsi du contexte aux œuvres particulières puis à des thématiques transversales dans un parcours bien ordonné. Mais au-delà de cette répartition classique, il apparaît rapidement que chaque contribution s'inscrit dans une analyse globale de l'œuvre de Xénophon et ne laisse jamais de côté les questions de la réception et des lectures modernes de celle-ci. La première section, "Contexts ", situe Xénophon et son œuvre dans son époque (I. "X. and his Times ", par John W.I. Lee, 2. "X. and Greek Philosophy", par Louis-André Dorion, 3. «X. and Greek Political Thought», par Sarah Brown Ferrario et 4. « X.'s Place in Fourth-Century Greek Historiography », par Nino Luraghi). Ainsi, en conclusion d'un article tout en nuance, Lee offre une réflexion intéressante sur les différentes conceptions de la vie de $\mathrm{X}$., déterminées par l'époque et la culture de leurs rédacteurs: "Every generation writes its own Xenophons» (p. 35) et souligne, comme il se doit, que ce sont les textes, plus que leur auteur, qui s'offrent aujourd'hui à l'analyse. Semblablement, Luraghi conclut son remarquable article sur la place de X. dans l'historiographie $d u \mathrm{IV}^{\mathrm{e}}$ siècle par une réflexion la réception de X. chez les auteurs postérieurs comme penseur ou philosophe, plutôt que comme historien. La seconde section, «Individual Works », traite systématiquement des différentes œuvres de X. (5. “X.'s Anabasis and Hellenica", par John Marincola, 6. “X.'s. Apology and Memorabilia», par David M. Johnson, 7. “X.'s. Symposium», par Gabriel Danzig, 8. “X.'s Oeconomicus », par Fiona Hobden, avec un intéressant appendice prosopographique sur la femme d'Ischomaque, 9. “X.'s Cyropaedia: Tentative Answers to an Enigma », par Melina Tamiolaki et Io. “X.: the Small
Works », par John Dillery). Je voudrais spécialement mettre en avant ici la contribution de Dillery qui, bien loin de donner un chapitre descriptif - passage obligé - sur les œuvres “mineures » de $\mathrm{X}$., offre une réflexion intéressante sur l'idée même d'œuvres mineures dans la réception moderne de l'œuvre de X. et sur les genres littéraires qu'elles illustrent. Les parties 3 et 4 sont à proprement parler les plus transversales. Les articles regroupés sous le titre "Techniques» s'intéressent très spécifiquement à la langue et aux techniques narratives de X. (II. "X.'s Language and Expression », par Vivienne Gray, I2. "X.'s Authorial Voice », par Christopher Pelling, I3. “Xenophon's Narrative Style », par Tim Rood et if. "The Character and Function of Speeches in X. » par Emily Baragwanath). Ces quatre contributions se distinguent par l'ampleur des questions traitées et leur très grande clarté, malgré une relative technicité. Place y est faite surtout aux grandes œuvres narratives (Cyropédie, Anabase, Helléniques), mais pas uniquement, et le lecteur peut s'y familiariser aussi bien avec les caractéristiques récurrentes du style de X. qu'avec les traits propres à certains textes. Les cinq contributions suivantes s'attachent à une série de “ Major Subjects » qui concernent, essentiellement, X. comme historien et théoricien de la politique (I5. “X. as a Historian », par Michael A. Flower, 16. “X. on Leadership: Commanders as Friends ", par Richard Fernando Buxton, 17. “X. and Athens », par Christopher Tuplin, I8. “X. on Persia », par Kostas Vlassopoulos et I9. “X.'s Views on Sparta» par Paul Christesen). Ces diverses contributions brassent nécessairement large, mais sans superficialité. Paul Christesen par exemple souligne bien la difficulté à traiter en une vingtaine de pages d'un sujet aussi complexe et controversé que la relation de X. à Sparte et aux Spartiates. Mais précisément, c'est en présentant de manière nuancée les différentes orientations de la 
recherche moderne et en les confrontant aux textes qu'il donne au lecteur le meilleur instrument pour se familiariser avec le sujet. La dernière partie, enfin, concerne la réception de l'œuvre de X. dans l'Antiquité (20. “ X.'s Influence in Imperial Greece », par Ewen Bowie) et aux époques moderne et contemporaine (2I. " $\mathrm{X}$. and the Instruction of Princes » par Noreen Humble et 22. «X.'s Changing Fortunes in the Modern World », par Tim Rood). On apprend notamment dans l'article de Noreen Humble comment la Cyropédie passe de source d'inspiration pour les specula principum à un modèle éducationnel pour l'honnête homme, avant d'être supplantée par l'Anabase dans le système scolaire à partir du $\mathrm{xIX}^{\mathrm{e}}$ siècle. L'épilogue rédigé par Edith Hall (“ X.: Magician and Friend ») clôt parfaitement ce parcours en revenant sur l'influence persistante de X. et le charme exercé par son œuvre sur ses lecteurs.

Ce livre est une remarquable introduction à $\mathrm{X}$. Chaque contribution est suivie d'un essai bibliographique, plus ou moins bref, et presque toujours limité à l'essentiel. Quatre cartes donnent, malgré un format nécessairement réduit, les repères géographiques indispensables et un tableau chronologique reprend, en fin de volume, les dates importantes dans la vie de X. Mais c'est l'index analytique, surtout, extrêmement détaillé, qui apporte à l'ouvrage une valeur supplémentaire en invitant le lecteur à se construire à son tour sa propre vision de Xénophon.

Olivier GENGLER Académie des Sciences de Heidelberg Université de Tübingen ogengler@yahoo.fr
Rebecca Futo Kennedy (dir.), Brill's

Companion to the Reception of Aschylus, Leyde et Boston, Brill, 2018, 634 p., I93 € / ISBN 9789004249325 (hardback) ou 9789004348820 (e-book).

Cet ouvrage collectif se compose de vingt-cinq articles, écrits en anglais, répartis en deux catégories inégales. La première, intitulée un peu étrangement "Pre-Modern receptions ", traite de fait de la réception d'Eschyle dans l'Antiquité classique et tardive : dans la Sicile antique ; dans la comédie ; chez Aristote ; à l'époque hellénistique; dans l'Empire romain et enfin à Byzance.

La seconde partie, “ Modern receptions », fait commencer cette modernité au $\mathrm{XVII}^{\mathrm{e}}$ siècle et suit ensuite l'ordre chronologique. C'est d'abord une étude sur l'opéra, dont la plus grande part est néanmoins consacrée à Wagner, Fauré et Carl Orff. De même l'article consacré à Eschyle et l'Allemagne met-il l'accent sur Wagner, entre la fin des Lumières et l'époque contemporaine. Wagner sera encore l'objet d'un article qui lui est entièrement consacré un peu plus loin, sous le titre « Form and Money in Wagner's Ring and Eschylean Tragedy ». Le $\mathrm{xIx}^{\mathrm{e}}$ siècle est en outre illustré par un auteur grec, Stephanos Demetriades, qui s'inspire du grand ancêtre pour une pièce intitulée Xerxes, dans une langue à mi-chemin du démotique et du grec savant; c'est ensuite l'Angleterre des Shelley, avec le Prometheus Unbound de Percy Bysshe, et le Frankenstein, or the Modern Prometheus de son épouse Mary. On retrouve le roman avec Vanity Fair de W. M. Thackeray et plus particulièrement le passage consacré à la bataille de Waterloo, toile de fond comparable à ce qu'est la guerre de Troie dans L'Orestie. C'est enfin une étude intéressante de représentations de drames d'Eschyle en langue originale à l'époque édouardienne, fin $\mathrm{XIX}^{\mathrm{e}}$ et début $\mathrm{Xx}^{\mathrm{e}}$ siècles. Une contribution plus technique s'intéresse à la question des itérations de 
mots dans Les Perses et des commentaires qu'elles ont suscités.

De là on saute allègrement à l'époque contemporaine, avec des réécritures et adaptations pour la télévision : une en France (1961) avec pour toile de fond la guerre d'Algérie, deux en Angleterre (1961 et 1979), la première destinée à un public adolescent, la seconde, The Serpent Son, visant un spectacle "primitif, barbare, exotique et ritualisé ». La politique est plus directement conviée avec deux films, celui de Pasolini, Appunti per un Orestiade Africana, et celui de Sissako, Bamako. Suit une étude consacrée à la réception des drames d'Eschyle en Afrique du Sud, pendant et après l'apartheid. C'est ensuite l'Amérique latine, notamment au Mexique et à Cuba. L'Amérique du Nord est présente avec O'Neill (Mourning becomes Electra, 1931) et le film de Werner Herzog (My Son, My Son, What Have Ye Done?, 2009) sur un script de Herbert Golder. De nouveau le cinéma avec Stanley Kubrick, The Shining (1980) comparéà Agamemnon, puis le roman de science-fiction avec Dune de Frank Herbert (1965). L'ouvrage se clôt avec deux articles de synthèse, l'un qui, au rebours de certaines contributions précédentes, s'étonne de l'absence d'Eschyle dans la réflexion politique moderne, l'autre qui analyse la réception moderne de la théorie politique eschyléenne telle qu'elle transparaît à travers ses drames.

Au total, un ouvrage où, sans aucun doute, il y a beaucoup à apprendre, mais qui présente aussi les défauts des ouvrages collectifs, partiels et partiaux. Aucun mot, par exemple, des traductions d'Eschyle par Paul Claudel ou Viatcheslav Ivanov au début du xxe siècle. L'introduction d'à peine quatre pages, intitulée “The Reception of Æschylus » ne comble certes pas cette faille: on aurait attendu une synthèse riche et systématique sur le sujet et l'état actuel des recherches. L'index, qui mêle noms propres, noms mythologiques, titres et “ thèmes » (sans qu’y figure néanmoins, comme on peut souvent le déplorer aujourd'hui, le contenu des notes !) trahit le caractère quelque peu superficiel de l'ouvrage. Mais chaque article est suivi d'une bibliographie de quelques pages: on a ainsi les moyens de poursuivre la recherche, en tout cas dans les domaines choisis par les auteurs et l'éditeur du livre.

$$
\begin{array}{r}
\text { Dominique Millet-GÉRARD } \\
\text { Sorbonne-Université } \\
\text { Dominique.mg@wanadoo.fr }
\end{array}
$$

\section{Antonio Gonzales et Maria Teresa Schettino (dir.), Les sons du pouvoir des autres. Actes du troisième colloque SoPHiA, 27-28 mars 20I4, Strasbourg, Institut des sciences et techniques de l'Antiquité (ISTA), 20I7, I 46 p., I9 €/ ISBN 97828486760o5.}

L'ouvrage constitue la publication des actes du troisième colloque SoPHiA tenu à Strasbourg en 20I4. Le titre manifeste ainsi la volonté de poursuivre la réflexion engagée quelques années plus tôt lors du colloque de Besançon, publié depuis (M. T. Schettino et S.Pittia [dir.], Les sons du pouvoir dans les mondes anciens, Besançon, 2012). En préambule, A. Gonzales et M. T. Schettino expliquent ce qu'il faut entendre par "autres»: “les groupes d'individus organisés ou non, qui étaient étrangers au monde gréco-romain ou qui [...] ont perturbé le bon fonctionnement des institutions » (p. 9). Ils défendent cependant untravail historique et non anthropologique, s'appuyant sur les sources classiques et ayant toujours en ligne de mire la culture gréco-romaine et le rapport entre pouvoir et bruits. Cette lecture "croisée " plutôt que “ comparée » (p. Io) était inévitable en raison du faible nombre de contributions. Ainsi, les huit articles, écrits à parité en italien et en français, suivis chacun par une bibliographie, sont organisés en trois 
parties: I. Les dissonances populaires; II. Bruits et représentations sonores du palais; III. Clameurs aristocratiques et guerrières.

Tout d'abord, J. Thornton montre que Polybe et Cicéron insistaient de manière caricaturale sur le vacarme des assemblées grecques et romaines et l'attribuaient à leur composition ethnique et sociale. Derrière cette clameur, ou même le silence imposé par la force, c'était l'incompétence des masses, leur irrationalité et les pressions qu'elles exerçaient que dénonçaient Cicéron et Polybe qui critiquaient la démocratie et les démagogues. Mais lorsque les cris ou leur absence étaient recherchés comme signe de respect ou d'approbation, la foule était désignée cette fois comme populus. L'interprétation des bruits de l'assemblée était donc un enjeu des débats politiques. A. Galimberti contextualise ensuite les complots contre Commode rapportés par Hérodien. Il s'intéresse au jeu entre le silence, des conjurés mais aussi de l'empereur lorsqu'il faisait disparaître ces derniers, et le tumulte et parfois même la théâtralité de l'attentat et de sa répression qui recherchaient l'un comme l'autre l'assentiment du peuple-témoin. A. Jacquemin nous fait ensuite visiter le sanctuaire de Delphes en relevant les bruits qui en troublaient la quiétude. Le paysage sonore était marqué par les cris des animaux qui vivaient là ou qui y étaient sacrifiés, mais aussi, selon l'heure, par les discours des guides pour ceux qu'on n'appelait pas encore “ touristes », les conversations liées aux banquets et aux activités politiques (le Conseil et le synedrion étaient situés dans le sanctuaire), et les bruits des athlètes qui s'entraînaient. Même en dehors des concours, le sanctuaire était un lieu plein de vie dans lequel le calme était parfois dur à trouver.

Dans la seconde partie, C. Mora tente de retrouver les intrigues et l'ambiance feutrée des palais du Proche-Orient à partir des serments, des édits et des correspondances puis L. Troiani se penche sur le monde hébraïque d'époque gréco-romaine, dans lequel les manifestations sonores servaient à rassurer ou à signifier l'éloignement et la stabilité du pouvoir, voire, pour les gouvernés, à inciter à la médiation.

G. Traina inaugure la troisième partie par une étude des tambours parthes qui sonnent le glas de Crassus à la bataille de Carrhes. Ces instruments, qui s'opposent aux tuba romains, seraient issus d'une tradition chamanique évoquant le dionysisme. Dans son récit, Plutarque inverse les rôles, présentant les Romains comme désordonnés et terrorisés par l'harmonie des percussions barbares. B. Pichon prolonge l'enquête en passant en revue les discours, les cris, les rires et les larmes, puis la musique et le chant chez les Gaulois avant la conquête. Ces « sons », qui servaient surtout aux auteurs gréco-romains à dramatiser leur récit, n'étaient pourtant pas si exotiques, et, codifiés, ils servaient à accroître le prestige des chefs. Le volume s'achève avec l'étude d'U. Roberto sur les cris, chants et danses de guerre que les Germains exécutaient pour se donner du courage, garantir la discipline, et qui pouvaient apparaître comme un présage pour l'issue de la bataille. Les chefs de guerre montraient l'exemple et retiraient du prestige de leurs talents. Si ces pratiques étaient largement partagées dans le monde archaïque, elles étaient devenues inconnues des légions impériales et ne s'y diffusèrent que durant l'Antiquité tardive à la suite du recrutement de barbares. Le cantator byzantin tirerait ainsi son origine du maître de chœurs germain.

On peut regretter deux absences: celle d'un index, et, celle d'une conclusion. Cela est d'autant plus dommageable que les termes du titre demeurent relativement flous à l'issue de la lecture du volume: le peuple entre-t-il vraiment dans la catégorie des « autres »? Les calomnies, les délations ou même les discours sont-ils des « sons »? Par ailleurs, étant le second volume con- 
sacré à la thématique, le caractère novateur se trouve logiquement affadi et on s'étonne d'autant plus du refus d'une majorité des contributeurs de recourir non seulement à l'anthropologie, mais aussi à l'histoire des perceptions, des techniques ou même des émotions. Tout cela débouche sur une histoire (trop) classique, certes intéressante, mais dans laquelle la dimension sonore apparaît comme un angle d'attaque parfois un peu artificiel.

Clément BuR Institut National Universitaire Champollion (Albi) clement.bur@univ-jfc.fr

Christa Gray, Andrea Balbo, Richard M. A. Marshall et Catherine E. W. Steel (éds.), Reading Republican Oratory.

Reconstructions, Contexts, Receptions, Oxford, Oxford University Press, 20I8, 366 p., £84 / ISBN 9780I9878820I.

Cet ouvrage est la publication des actes d'un colloque qui s'était déroulé les I5-I7 avril 2015 à Turin dans le cadre du projet ERC Fragments of the Roman Republican orators. L'introduction explique le cœur du projet et la méthodologie adoptée. Un des enjeux est de mettre en évidence l'art oratoire au-delà de l'omniprésent Cicéron, de s'intéresser aux fragments des discours perdus et à l'éloquence féminine notamment. L'approche, résolument diachronique, cherche à mettre en lumière la spécificité de l'éloquence républicaine, y compris dans sa réception impériale. Les dix-neuf contributions sont ainsi réparties dans deux parties : la première concernant la transmission aux époques républicaine puis impériale; la seconde étant plutôt consacrée à la reconstruction des fragments dans leur contexte politico-social.

Les quatre contributions de la première partie sur la transmission intègrent les problématiques d'élaboration de l'éloquence et des discours normatifs, mais aussi celles de sélection et de réception: au contact d'abord de la Grèce dans un contexte d'impérialisme romain et d'attrait pour la paideia (A. Eckert, "Roman Orators between Greece and Rome: The Case of Cato the Elder, L. Crassus, and M. Antonius »). L'impact des influences interculturelles et de la culture étrangère est également analysé par I. Goh à travers l'exemple de Lucilius («Republican Satire in the Dock: Forensic Rhetoric in Lucilius »). L'éloquence diplomatique est étudiée par E. Torregaray Pagola grâce à l'Amphitruo de Plaute mettant en lumière des pratiques diplomatiques des legati au $\mathrm{II}^{\mathrm{e}}$ s. av. J.-C., comme la rhétorique coercitive et l'intimidation («Plautus and the Tone of Roman Diplomacy of Intervention »). Il est difficile d'apprécier les qualités oratoires de P.Sulpicius Rufus et de G.Aurelius Cotta tant le jugement de Cicéron est incontournable, puisqu'il n'existe pas de témoignage direct. A. Casamento propose cependant de revenir sur ces figures («The Eloquence of Publius Sulpicius Rufus and Gaius Aurelius Cotta in Cicero's Brutus »).

L'époque impériale connaît une mutation profonde de l'art oratoire qui cesse d'être un instrument essentiel de la communication politique.Les auteursimpériaux ont doncun point de vue différent, même si l'éloquence perdure dans la culture romaine. C'est le cas de Quintilien. Ainsi A. Raschieri souligne-t-il l'hétérogénéité des fragments collectés par Quintilien à des fins diverses, par exemple pour illustrer des points de grammaire ( The Fragments of Republican Orators in Quintilian's Institutio oratoria»). Quintilien cite surtout des orateurs de la fin de la République et de l'époque triumvirale comme M. Valerius Messalla Corvinus (cos. 3I). S. Lawrence met ensuite en évidence l'opinion négative de Valère Maxime sur le pouvoir de l'éloquence dans la vie politique. Cette potentia est une source de danger et peut corrompre la 
liberté des citoyens («Vis and Seruitus: The Dark Side of Republican Oratory in Valerius Maximus »). C. Burden-Strevens invite ensuite à relativiser la pure invention des discours républicains dans l'œuvre de Dion Cassius, car l'historien a non seulement conservé certains contenus, mais aussi des stratégies rhétoriques (“Reconstructing Republican Oratory in Cassius Dio's Roman History »). Enfin J. Dugan s'intéresse à un orateur et poète méconnu du $\mathrm{II}^{\mathrm{e}} \mathrm{s}$. av. J.-C., G. Titius, et sa réception dans l'œuvre tardive de Macrobe, y compris dans une perspective d'anthropologie culturelle ( Netting the Wolf-Fish: Gaius Titius in Macrobius and Cicero »).

La deuxième partie consacrée à l'étude des fragments de l'éloquence républicaine dans leur contexte politico-social est organisée en plusieurs thématiques. D'abord l'analyse porte sur la reconstitution des fragments dans un contexte littéraire. A. Cavarzere se focalise alors sur l'étude de cas de G. Titius permettant de reconstruire son œuvre rhétorique fragmentaire (“ Gaius Titius, Orator and Poeta. [Cic. Brut. I67 and Macrob.Sat. 3.I6.4-16]»). Puis A. Corbeill s'intéresse à un personnage bien plus connu, Clodius, et tente de reconstituer un de ses discours perdus concernant une décision des haruspices en 56 (“Clodius'Contio de haruspicum responsis »). Le tribun de la plèbe de 58 av. J.-C. est aussi au cœur de l'article suivant. K. Morrell essaie de reconstituer les débats entre Cicéron et Caton sur la validité des décisions prises par Clodius ( "Certain gentlemen say...": Cicero, Cato, and the Debate on the Validity of Clodius' Laws ").

Les articles suivants mettent plutôt en avant la performance oratoire. J. Hilder insiste sur le caractère didactique de la Rhétorique à Herennius permettant aux apprentis orateurs de développer leurs compétences, y compris en tenant compte de la gestuelle et de la théâtralité (“The Politics of Pronuntiatio: The Rhetorica ad Herennium and Delivery in the Early
First Century BC »). Dans une perspective diachronique, A. Balbo s'intéresse à l'actio des orateurs, c'est-à-dire à l'ensemble des effets vocaux, gestuels et plus largement au langage corporel dans les fragments conservés dans les $O R F$ et FRRO (« Traces of Actio in Fragmentary Roman Orators »). C. Rosillo-López traite ensuite la question de la délimitation de l'éloquence dans la communication politique et examine dans quelle mesure des conversations informelles peuvent être considérées comme des témoignages fiables des discours publics ( I I Said, He Said: Fragments of Informal Conversations and the Grey Zones of Public Speech in the Late Roman Republic »).

La dernière partie s'intéresse notamment à l'éloquence funéraire, à la sphère féminine et aux gender studies. H. Beck étudie un moment hautement ritualisé, celui des funérailles aristocratiques, l'étude met en avant les émotions, les normes culturelles et leur mise en scène (“Of Fragments and Feelings: Roman Funeral Oratory Revisited»). L'éloge funèbre n'est pas réservé aux hommes comme le montre C. Pepe («Fragments of Epideictic Oratory: The Exemplary Case of the Laudatio Funebris for Women »). Le rôle de Fulvie dans les funérailles de son époux Clodius est ensuite mis en avant par B. Gladhill, notamment la mise en scène de son désespoir, les ruptures avec le rituel classique et l'irruption sur la scène politique ("Women from the Rostra: Fulvia and the Pro Milone »). Enfin la figure de Cornelia est réévaluée par J. Hallett («Oratorum Romanarum Fragmenta Liberae Rei Publicae: The Letter of Cornelia,Mater Gracchorum, and the Speeches of her Father and Son »).

En fin d'ouvrage le lecteur trouvera une bibliographie conséquente et actualisée (p. 3rg-354) et des index. Pour conclure, l'ouvrage offre des études de cas nombreuses et détaillées. L'approche rigoureuse et renouvelée de l'éloquence 
républicaine offre ici un volume essentiel pour quiconque souhaite travailler sur cette thématique.

Cyrielle LANDREA

Université Bretagne Sud cyrielle.landrea@univ-ubs.fr

Tanja Itgenshorst et Philippe Le Doze (dir.), La norme sous la République et le Haut-Empire romains. Élaboration, diffusion et contournements, Bordeaux, Ausonius, 20I7, 68г p., 3о є/ ISBN 9782356ı3ı 805.

Dès la première phrase de l'Avant-propos, T. Itgenshorst et P. Le Doze rappellent que la norme " recouvre des réalités complexes et se révèle être une notion plurielle» (p. II). Ils donnent ainsi ce qui fut le ton du colloque international de 2014 dont est issu cet ouvrage : une (trop?) grande ouverture thématique, dans un arc chronologique assez ample. Sont ainsi envisagés la nature des normes (avec le choix de ne pas se limiter au droit), leur application, leur élaboration, leur rôle dans la construction de l'individu et du groupe, et leur évolution.

Un objectif important du colloque était de relancer le dialogue franco-allemand en histoire romaine autour de la normativité. Ce thème est effectivement d'autant plus pertinent qu'il est au cœur des deux traditions historiographiques depuis plus d'un siècle. Loin d'être épuisée, cette question connaît même un renouveau comme en témoigne la récente élection de D. Mantovani à la chaire "Droit, culture et société de la Rome antique » du Collège de France. Notons qu'une entrave bien connue à cette collaboration, et qui ne fait pas gloire à la France, transparaît dans cette publication: seuls deux collègues allemands sur sept écrivent dans leur langue. On peut toutefois espérer que cela contribuera à diffuser, notamment auprès des étudiants, les travaux de chercheurs outre-Rhin de premier plan.

Une des forces de cet ouvrage est ainsi de proposer une solide introduction théorique composée de trois articles. Le premier de C. Lundgreen offre un salutaire effort de définition, rappelant que la norme est d'abord une construction sociale que l'on peut transgresser et qui est plus ou moins institutionnalisée. Il défend ainsi deux distinctions heuristiques entre règles et principes (en prenant l'exemple du droit au triomphe) et entre collision de normes et concurrences de normes. L'exploration d'H. Bruhns offre une réflexion sur un aspect de la pensée webérienne, souvent utilisée en histoire ancienne. En revanche, le comparatisme proposé par D. Engels, quoique fondé sur une idée stimulante, est décevant car il se limite à un survol peu étayé sinon par de longues citations. La conclusion de C. Badel fait pendant à cette réflexion liminaire et insiste à bon escient sur le mos maiorum que l'on rencontre dans la plupart des contributions et sur le choix du terme contournement plutôt que transgression.

Il est bien entendu impossible de résumer la trentaine d'articles répartis dans les huit parties qui feront le bonheur de chaque spécialiste (introduction, naissances des normes, religions, rôle des institutions et des élites, rôle du peuple, l'armée, la diffusion, conclusion). Nous nous contenterons donc d'attirer l'attention sur quelques contributions qui ont suscité notre intérêt.

J.-M. David aborde la construction de la norme par le biais des déclamations. Ces exercices de rhétorique permettaient de discuter dans un cadre privé de la conduite à adopter, notamment lors des guerres civiles, avant de se cantonner à une dimension esthétique sous l'Empire. F. Hurlet part d'un épisode méconnu du début du Principat d'Auguste : sa tentative de passer à trois consuls. Il démontre ainsi la force de la règle de la dualité au sommet 
de l'État et l'impossibilité d'imposer une nouvelle norme malgré des transgressions préalables nombreuses. C. Moatti offre un avant-goût de son livre paru depuis (Res publica: histoire romaine de la chose publique, Paris, 20I8) en étudiant comment la défense de la res publica émergea comme principe avant de devenir une norme légale et favorisa ainsi la conceptualisation même de la chose publique.

É. Deniaux s'empare de la question de la concurrence entre les normes en montrant que les jeux, que les édiles se devaient d'offrir au peuple, rendaient les règles de l'amitié plus puissantes que celles du bon gouvernement, à l'instar de Cicéron se résignant à demander à ses amis de Cibyra de capturer des panthères pour les chasses que son ami M. Caelius Rufus allait donner. E. Flaig examine également la tension entre l’habitus sénatorial de la conciliation et l'intransigeance liée aux ambitions personnelles à travers le cas paroxystique des tribuns de la plèbe qui avaient les moyens de bloquer la vie politique romaine. Son analyse du veto tribunicien de I86 contre un triomphe montre comment l'explicitation des normes de conduite de la classe dirigeante déboucha in fine sur des transgressions. Le cas examiné des normes vestimentaires par J. Meister est également éclairant : la toge fut peu à peu abandonnée dans le cadre privé mais se maintint dans l'espace public, devenant le costume exigé pour les cérémonies officielles sous l'Empire.

L'article collectif sur la religion pose d'intéressantes questions, notamment sur la transmission des normes par une personne et non un texte. Cette « humanisation » de la norme lui offre une capacité d'adaptation qui est d'autant plus efficace qu'elle encadre la pratique rituelle. L'importance de la pratique et des stratégies transparaît aussi dans l'étude que R. Baudry consacre aux témoins lors de la rédaction des sénatus-consultes, dossier souvent négligé et qui offre pourtant d'intéressantes pistes de réflexion : le choix se faisait-il selon la dignitas? la spécialisation? ou résultait-il des négociations entre sénateurs?

La question du rôle du peuple dans l'élaboration de la norme est abordée dans deux articles. T. Lanfranchi le limite aux contiones qui servaient de test aux rogationes permettant de les amender. Selon lui, les assemblées ne gagneraient en importance que quand la compétition aristocratique se dérèglait et que le Sénat ne parlait plus d'une voix unie. Le recours au peuple comme ultime arbitre découlerait de sa souveraineté et permettrait de dépasser la querelle historiographique autour de la nature de la République romaine. Quant aux comices curiates, étudiés par F. Van Haeperen, ils préservaient la pax deorum et le nouvel équilibre social et politique en actant des changements dans les familles (adoption, testament) et dans les collèges de prêtres et de magistrats.

L'étude de la diffusion des normes fait l'objet d'une approche originale quant aux sources retenues. A. Suspène rappelle ainsi combien les monnaies étaient le produit de normes techniques contraignantes, et néanmoins fluctuantes (sans compter les transgressions servant à se distinguer), au point d'être un élément constitutif de la romanité. De la sorte il souligne aussi leur importance pour exprimer la mémoire de la cité et ainsi en véhiculer le système de valeurs, en particulier l'éthique aristocratique. Enfin la monnaie servait à organiser la communauté puisque les censeurs classaient les citoyens selon des critères censitaires et que la solde versée solennellement aux soldats attestait leur qualité de citoyen. M. Corbier se penche ensuite les graffitis, écriture qu'elle qualifie de libre et néanmoins normée quant au support, au contenu (les devinettes pour trouver le nom de la femme aimée) et à l'emplacement (ne pas écrire sur un décor ou sur un précédent graffiti) comme en témoigne leur caractère répétitif. U. Walter, contre D. Mantovani, retourne à une vision 
de la loi comme simple instrument de communication politique en raison des difficultés à l'appliquer et de son caractère souvent temporaire. Il souligne à juste titre que toutes les grandes lois réorganisatrices furent votées dans des contextes de violence ou de pouvoir personnel.

Malgré l'effort initial de définition, les contributions ont chacune adopté une interprétation extensive de la norme, entravant ainsi les tentatives de confrontation. L'absence du droit pénal et privé, qui est pourtant un champ d'études fécond, est également dommageable pour une réflexion qui se voulait la plus large possible. Le volume offre ainsi une série d'études juxtaposées débouchant sur une sorte de tour d'horizon de la question, malgré la qualité des contributions, tout particulièrement des introduction et conclusion dont la lecture est très stimulante.

Pour finir saluons la présence de deux index (sources et noms) et d'une abondante bibliographie générale, faisant de ce riche recueil une lecture et un outil nécessaires à tout historien de la République romaine.

Clément Bur

Institut National Universitaire Champollion (Albi) clement.bur@univ-jfc.fr

Hans Kopp et Christian Wendt (éds.), Thalassokratographie. Rezeption und Transformation antiker Seeherrschaft, Berlin, De Gruyter, 20I8, 3i6 p., 79,95 € / ISBN 9783пго568899, ISSN ı8645208.

Et si le lecteur commençait par la fin? Dans ce cas, il visiterait des parcs d'attractions à thème dans différents pays européens, revivrait des aventures d'Ulysse et en sortirait, trempé peut-être, mais fortement impressionné par la dangerosité de la Méditerranée et la "bravery in dominating the seas » (p. 298) du héros grec.
Laissons la question ouverte de savoir si Ulysse a dominé la mer ou si ce n'est pas plutôt l'inverse et revenons au début du livre où les directeurs de cette publication expliquent le néologisme qu'ils ont choisi pour titre. Ce n'est pas la "Seeherrschaft» (thalassocratie, imperium maris, dominium maris) dans sa dimension politico-militaire à certaines époques de l'Antiquité et sa “ réception" dans l'historiographie ultérieure qui sont l'objet de l'enquête, mais au contraire la réflexion, et les traces qu'elle a laissées, sur un phénomène beaucoup moins univoque, beaucoup plus diffus, où se mêlent des dimensions politiques et stratégiques avec des discours de nature légitimatrice (la domination), de recherche identitaire ou de nature polémique avec des débats juridiques ou encore avec des aspirations à dominer le monde entier. Montrer l'enchevêtrement entre une multitude et grande variété de “ discours », au sens très large, sur la thalassocratie, d'une part, la réception de l'Antiquité d'autre part, est l'ambition affichée ici. Renoncer ainsi à une définition étroite de l'objet signifie une grande liberté pour les contributeurs qui, c'est la loi du genre, n'ont pas forcément exactement la même vision que ceux qui ont organisé le colloque berlinois de 2015 et composé ce recueil.

Mais c'est tout bénéfice pour le lecteur. S'il fait l'effort de lire la longue introduction, il a à sa disposition des instruments de méthode et de compréhension qui lui permettent de profiter doublement de la richesse de la douzaine de chapitres, dont la moitié en anglais: on peut les lire de façon " naïve ", sans les relier aux réflexions méthodologiques exposées dans l'introduction, mais aussi à travers les lunettes réflexives proposées par Hans Kopp et Christian Wendt. Les deux premiers chapitres en sont de bons exemples: Hartmut Böhme étudie la « transformation » moderne de représentations antiques de l'océan chez Christophe Colomb et Alexander von Humboldt, tandis que 
Christian Wendt analyse la relation homme-mer dans l'Antiquité et sa réception ultérieure. L'analyse de la réception se transforme ici en une recherche sur de nouvelles perspectives permettant de comprendre autrement les phénomènes antiques.

Les regards ambivalents de Rome et de l'Angleterre (dans les années I732-I759) sur la domination maritime athénienne sont étudiés par Ernst Baltrusch et Ben Earley. Le cas anglais est d'autant plus intéressant que c'est John Dee, entre autres conseiller en science et en astrologie de la reine Elizabeth, mais aussi conseiller en navigation lors des grandes découvertes et inventeur du terme Empire Britannique, qui, vers la fin $\mathrm{du} \mathrm{xvI}^{\mathrm{e}}$ siècle, introduit le terme antique thalattocratia dans le débat politique moderne, empruntant l'expression à Strabon chez qui elle apparaît pour la première fois dans l'Antiquité. Ce chapitre de Hans Kopp sur John Dee et la redécouverte de la thalassocratie au $\mathrm{XVI}^{\mathrm{e}}$ siècle montre bien la différence entre une approche philologique qui retrace l'histoire des mots dans des textes et traductions, d'une part, et d'autre part la mise à jour d'un concept, sa réinterprétation, sa transformation et son introduction dans un contexte nouveau. La contribution de Louis Sicking sur les Pays-Bas habsbourgeois étend le spectre des thèmes abordés au-delà des aspects symboliques et de représentation inhérents aux évocations de l'Antiquité. Pour être souverain de la mer, de quelle flotte l'Empereur a-t-il besoin : de navires loués en cas de besoin ou achetés pour constituer une flotte permanente? Quels impôts pour la payer, quelle finalité : la sécurité de la mer pour le commerce?

Au tournant du XvI ${ }^{\mathrm{e}}$ au XvII ${ }^{\mathrm{e}}$ siècle, c'est le droit romain, comme nous le rappelle Kaius Tuori, qui est mobilisé par Hugo Grotius (Mare Liberum, i6og) dans l'intérêt de la Compagnie néerlandaise des Indes orientales dans son conflit avec le Portugal et l'Espagne, défendeurs, comme l'Angleterre, de la conception du mare clausum, pour soutenir la liberté de commerce sur mer et l'exploitation libre des ressources de la mer. Pour les Romains la mer ne se trouvait pas dans le même régime juridique que la terre : "The limit of the sea was a limit of the law, it was common property of all mankind, meaning it could not be owned or controlled»(p. 2I/4). Grotius arrivait à la même conclusion par le biais d'une argumentation différente : c'est le droit privé romain qui réservait la mer, res nullius, à l'usage de l'humanité tout entière.

Dans la dernière section du livre trois chapitres sont consacrés à des discours très différents sur les dominations antique et moderne sur la mer. Barry Strauss traite de trois "modern naval thinkers ", deux amiraux américains du $\mathrm{xIx}^{\mathrm{e}}$ et du $\mathrm{xx}^{\mathrm{e}}$ siècle, ainsi que d'un géopoliticien angloaméricain contemporain. Dans les trois cas, les références aux dominations et stratégies navales antiques n'ont finalement qu'un intérêt limité. Joshua Derman s'intéresse dans une étude stimulante et bien documentée à deux penseurs de la géopolitique, deux juristes allemands de la période national-socialiste et du début de la guerre froide : Carl Schmitt et Ernst Wolgast, nés d'ailleurs la même année, I888. On connaît la vision schmittienne de l'histoire: une lutte permanente entre des puissances terrestres et maritimes. Moins connues sont les théories de Wolgast. Si pour Carl Schmitt la thalassocratie est surtout aussi un topos de sa polémique anti-anglaise, Wolgast part de l'exemple athénien et accorde, dans une analogie directe, à la thalassocratie britannique le rôle central dans un nouvel ordre maritime du monde.

Edith Foster, enfin, nous transporte du règne maritime d'un Périclès ou Thucydide vers les premières représentations de la domination sur les airs chez Giulio Douhet, théoricien italien de la guerre aérienne, vers la littérature de fiction d'un H. G. Wells et, en bouclant la boucle, vers Rex Warner, 
traducteur de La guerre du Péloponnèse (1954), qui, alors que la Luftwaffe de Hermann Göring bombarde Londres, met en scène dans son roman The Aerodrome: A Love Story (I94I) une incarnation moderne de Périclès : the Air-Vice-Marshal. Ici, la question de la réception-transformation fonctionne dans le sens inverse : la guerre moderne, la guerre aérienne menée par un type d'homme qui fonctionne selon une rationalité inédite, a-t-elle influencé la traduction et l'interprétation de l'œuvre de Thucydide par Warner?

Somme toute un livre riche et stimulant, bien fait, agréable à lire et à tenir entre ses mains. Un index des personnes, des concepts et lieux géographiques, ainsi que d'événements historiques marquants permet au lecteur de naviguer facilement sur les océans si variés présentés ici.

Hinnerk BRUHNS Centre de recherches historiques (EHESS/CNRS) bruhns@ehess.fr

\author{
Antonio La Penna, Io e l'antico. \\ Conversazione con Arnaldo Marcone, Pise \\ et Florence, Della Porta Editori, 20I9, 2I2 \\ p., I $5 € /$ ISBN 9788896209370 .
}

Readers of this remarkable little book would be well advised to start from its iconographical appendix. There is a widespread, antagonistic and comforting narrative that dominates much of the discourse on Classics as a subject, especially in English-speaking countries: that it is the domain of privilege and entitlement, the perquisite of sheltered and propertied minorities, the quintessential ivory tower. It takes a cursory glance at the photograph of the square of Bisaccia, the small town in Irpinia where Antonio La Penna grew up, to irreversibly problematise that picture. He was born in 1925; his mother was illiterate, his father a small farmer who enjoyed reading Tolstoy and Hugo in the evenings, whenever he was not too tired to do any reading. What enabled a boy from one of the most deprived communities in Southern Italy to become one of the most authoritative Latinists of the last century was access to free State education: first at the Liceo Colletta in Avellino, then at the Scuola Normale Superiore in Pisa, where he passed the entry examination at the prodigious early age of sixteen. By I956 he held a University post in Florence, after a few years of high school teaching on which he always looked back fondly; he taught there and in Florence for nearly forty years; he is still at work in his midnineties, attending to the final instalments of a collaborative commentary project on Sallust's Historiae.

In the first part of this book he has a wide-ranging conversation with Arnaldo Marcone (p. I3-8o), followed by three substantial appendices that make up the bulk of the volume (p. 8I-I9o). Marcone, a distinguished Roman historian, was a student of La Penna at the Scuola Normale, but not one of his immediate pupils. That gives the conversation a more lateral and less predictable outlook than one might expect to be the case in a book of this kind (a previous item of the series in which this volume appeared is Emilio Gabba's important Conversazione sulla storia with Umberto Laffi). La Penna tends to give brief, often self-effacing answers - and every single word matters. We do not get a linear intellectual autobiography; that may be found in the first appendix, the text that La Penna penned when he received the Premio Feltrinelli of the Accademia dei Lincei in I987 (p. 83-92): a terse and characteristically insightful piece, from which emerges the image of a modest man, who agrees to provide an account of the trajectory of his interests, but has little appetite for assorting the scale of his achievements and his centrality in much of the Roman studies in 
the second half of the twentieth century (cf. p. 85: 'la lunga attività di insegnamento... ha assorbito gran parte delle sue non molte energie'). In his conversation with Marcone, La Penna's modesty emerges even more strikingly: he even makes the point that a professor is almost constitutionally a man without biography (p. 76). Yet his interviewer ably identifies and brings out some focal points of interest, on which La Penna's biography sheds light: the profound debt to his Irpinian origins, both towards his family and towards a wider cultural milieu, in which literary criticism has never been decoupled from political engagement (one could draw a line from Francesco De Sanctis to Carlo Muscetta); a long-standing commitment to Marxism, both as a yearning for social justice and as a tool of historical analysis and interpretation; and the firm alliance (dating back to his school years and further developed through the teaching and example of Giorgio Pasquali) between linguistic and philological training and the exploration of literary problems - of what La Penna so aptly terms, here and elsewhere, 'il gusto'. The subtitle of the interview is 'Conversazione sulla filologia e sulla scuola', and honestly encompasses much of La Penna's core interests. The vast body of his writings on the problems of the Italian school system and of the prospects of the teaching of Classics languages and culture stands as a testimony to his wider civic commitment: the appendices, two pieces from 1999 and 1993 respectively (' $\mathrm{La}$ crisi della scuola media in Italia. Alcune proposte di riforma', p. 93-I49 and 'Noi e l'antico', p. I5I-I9o), are not just significant to a professional community: right at their core lies a certain idea of Italy (see esp. p. II8-II9 on 'la crisi morale del paese'), and an historical and political agenda that stands the test of time.

An assessment of La Penna's intellectual trajectory will be central to any attempt to understand the quality and range of Italian post-war classical scholarship, and indeed of its international impact (not least if one thinks of the trajectories of some of his pupils). This book is an important step towards that undertaking, which will surely have to be a collaborative one. Its most immediate and more profound reward, though, is to convey, in his own words, the image of a kind, unassuming, and straightforwardly great master of our studies. A man to whom scholarship, teaching, and civic engagement have been deeply integrated modes of the same intellectual commitment.

\section{Federico Santangelo \\ Newcastle University federico.santangelo@ncl.ac.uk}

Aude Lehmann (dir.), Diderot et l'Antiquité classique, Paris, Classiques Garnier, 2018, 388 p., 49 €/ ISBN 9782406073253.

Nos collègues de l'université de Mulhouse (groupés au sein de l'ILLE, Institut de recherche en Langues et Littératures européennes) à l'initiative d'Aude Lehmann, ont eu l'idée très pertinente, en cette année 2013 où l'on célébrait le Tricentenaire de la naissance du philosophe, d'organiser un colloque sur “Diderot et l'Antiquité classique » dont ce volume publie les actes. Fort pertinente en effet, que cette idée de sonder chez Diderot la présence, la connaissance et la pratique des Anciens, pour évaluer l'incidence de celles-ci sur sa philosophie «moderne», façon aussi de raviver pour la réconcilier la querelle du même nom. Cette question s'avère d'ailleurs tout aussi pertinente dans le cas des autres grandes figures des Lumières, Montesquieu, Voltaire et Rousseau.

La tâche s'avère toutefois ardue, même si Aude Lehmann, faisant appel au paradoxe souligné par Jacques Chouillet (Diderot, Paris, Sedes, I977, p. I03), présente avec 
beaucoup de finesse dans son introduction, à travers la formation du philosophe passé chez les Jésuites puis par la faculté de Théologie, tout ce qu'il doit à l'Antiquité : une dette qui apparaît beaucoup plus comme un tremplin pour les audaces de sa pensée, qu'une somme érudite dont il resterait tributaire. Serait-ce qu'il fut encouragé par des précurseurs tels Platon pour l'art du dialogue, ou Aristote, voire Horace pour leur capacité méta-discursive de théorisation des genres? Toujours est-il qu'il sait user avec discernement du génie des Anciens, séparant le bon grain de l'ivraie, traquant l'“anticomanie» de ses contemporains et reconnaissant, en Art, le caractère indépassable de l'esthétique grecque appelée à consolider sa théorie du “ modèle idéal ».

L'immensité du sujet explique peutêtre la complexité du plan thématique de l'ouvrage. Un autre type d'organisation aurait sans doute été possible (par époques dans la vie du philosophe, ou par genres littéraires), pour aborder méthodiquement l'imprégnation du philosophe par les grands auteurs de l'Antiquité. L'étude de Robert Bedon, placée en ouverture, avait-elle une valeur symbolique? Elle reprend à nouveaux frais l'apport de l'article LANGREs en matière de descriptions et de connaissances antiques. Elle révèle les sources utilisées par son auteur pour enrichir et embellir la ville natale de Diderot d'un passé galloromain bien réel mais discret, effacé par les reconstructions baroques de la contreréforme. L'attribution de cet article de l'Encyclopédie à Diderot lui-même reste toutefois, aux dires des spécialistes, problématique. Le débat reste ouvert.

La première partie proprement dite du volume (“Diderot critique d'art à l'école des Anciens ») s'ouvre sur le constat, à la lecture de certains de ses ouvrages, de la forte imprégnation du philosophe par le monde gréco-latin, sensible par exemple à travers les connaissances fines dont il témoigne dans les articles spécialisés qu'il supervise (АмphitheÂtre, Aqueduc), ou bien dans ses Salons qui manifestent des connaissances essentielles sur les œuvres antiques (les ruines d'Hubert Robert dans le Salon de I767). Jean-Marie André, dans “Diderot et l'antiquité romaine. Antiquités et Antiquité », explique le respect de ce savoir chez le philosophe par son “besoin d'historicité » qui exclut “ le culte superficiel des "antiquités" ». Dans le même esprit, Mary-Anne Zagdoun (“Diderot et l'esthétique d'Aristote ») montre en Diderot un lecteur attentif au théoricien antique dans la rédaction de son essai De la poésie dramatique, en ce qu'il y conserve à la lettre l'idée d'un théâtre rigoureusement pensé dans un but moral. Son goût pour la comédie sérieuse se déroulant dans un milieu moyen, l'action unique et conséquente, la vraisemblance, l'extraordinaire (et non le miracle), la simplicité et la sobriété, le port de l'habit de tous les jours chez les comédiens, il les doit à Aristote. Il reprend toutefois sa liberté de dramaturge en ce qui concerne la notion de caractère (qu'il conçoit, lui, comme malléable et évolutif, plus efficace aussi quand il s'oppose aux situations); la notion d'inspiration poétique, plus "spirituelle» que celle d'Aristote; enfin la notion de beauté, plus "morale» chez Aristote, plus “ mathématique » chez Diderot, car évaluée comme la perception d'un juste rapport entre le tout et ses parties. D'une façon plus concrète encore, Dominique Bocage-Lefèbvre étudie ce foisonnement de références antiques à travers les Essais sur la peinture, l'Éloge de Térence, les Pensées détachées sur la peinture («La célébration de l'Antiquité dans trois œuvres esthétiques de Diderot »), sans toutefois tirer, en conclusion, d'autre vision que celle, "kaléidoscopique ", d'une “mosaïque savante». C'est plutôt l'éclatement même de l'œuvre de Diderot qui devrait porter ces qualificatifs, et non la présence de l'Antiquité proprement dite, qui forme au contraire ce substrat sur lequel s'appuie une pensée en mouvement. 
Plus pertinentes selon nous sont les notations de la contributrice concernant l'emprunt à Térence d'une narratio souple, de la varietas chère à la rhétorique latine, ou la prégnance du dialogue socratique accordant une vive importance au lecteur ; l'influence des Pensées de Marc-Aurèle ou du Manuel d'Épictète sur l'écriture des Pensées détachées; enfin l'admiration pour l'homme Térence, esclave et écrivain, dont le statut social ne pouvait que susciter la sympathie d'un philosophe lui-même de modeste extrace. Fort riches également sont les deux textes suivants dus à Nadège Neumuller et Charles Philippe Assembé Ela, observant Pline l'Ancien comme l'une des sources essentielles, respectivement des Salons (« Diderot et l'histoire plinienne des arts ») et de la controverse avec Falconet ( Un paragone oublié : Diderot-FalconetPline »). Chez Pline, selon N. Neumuller, même conception que plus tard chez Diderot, de l'ekphrasis comme pratique affranchissant l'écrivain de l'éloge ; même attention à l'égard des grands modèles comme étalons d'une beauté liée aux proportions (le Laocoon, l'Hercule au repos - l'Hercule Farnèse). Selon Ph. Assembé Eba, c'est bien en s'appuyant sur le modèle plinien que Diderot, contrairement à Falconet, ose s'affranchir d'une critique d'art technicienne pour aller vers une forme poétique en soi.

Dans la deuxième partie, “Diderot et les philosophes de l'Antiquité », c'est à nouveau le dramaturge qui est interrogé dans une communication de Sophia Felopoulou. Celle-ci souligne la tension entre un "Rousseau platonicien» et un Diderot “aristotélicien », qui privilégie la fonction sociale et utilitaire du théâtre. Mais l'auteure montre de façon sensible comment l'intention morale plus encore que la forme l'emporte, faisant de Diderot, à travers le paratexte de son théâtre et ses textes critiques, un auteur politique résolument platonicien dans sa volonté de réformer la société ( Le dialogue diderotien avec Aristote et Platon »). Christine Hammann («Sénèque avocat de Diderot, procureur de Rousseau ») et Cécile Merckel (“La figure de Sénèque dans l'essai sur les règnes de Claude et de Néron ») montrent brillamment dans deux contributions complémentaires l'importance de Sénèque dans l'œuvre tardive du philosophe. La première évoque la fascination des deux frères ennemis, Rousseau et Diderot, et pour des raisons différentes, pour le même personnage de Sénèque, ce jeu de miroirs les rapprochant et les divisant à la fois. La seconde montre comment Diderot opposa et rapprocha habilement la vie et l'œuvre du moraliste antique pour justifier cette double vie qui fut la sienne, un pied dans le siècle, un pied en dehors, et le contemplant déjà de l'œil de la postérité. Dans une perspective appliquée à cette problématique, Valérie Perez montre subtilement, dans “Le jeu agonistique de la parole. Diderot et le parler-vrai », comment celui-ci, contrairement à ce que laisse supposer son texte ambigu sur Sénèque, eut pour véritable ambition lors de son voyage en Russie, de fléchir Catherine II dans le sens éclairé de sa philosophie politique, grâce à la technique du « parlervrai », cette parrhésia héritée de Dion (voir, chez Plutarque, la Vie de Dion) étudiée par Michel Foucault (Le gouvernement de soi et des autres, leçon de ig83 publiée en 2008).

Dans la troisième partie ( Diderot critique littéraire des Anciens »), Tatiana Smoliarova étudie de façon savante l'application que fit Diderot de son érudition et de sa virtuosité en matière de poésie hellénistique (notamment de l'œuvre difficile de Pindare et ses odes), produisant, selon le principe de la triade pindarique, les étonnants Eleuthéromanes dans l'intention carnavalesque de renverser cette forme d'éloge royal en chant burlesque et républicain (“L'inspiration pindarique chez Diderot ou la composition personnifiée »). Marie Saint-Martin, revenant encore sur la question du théâtre, montre astucieusement 
comment la critique du théâtre antique par Diderot fonctionne comme une "coquille vide » qui n'en retient que les idées et très peu les textes, s'inspirant ainsi de toute une tradition qu'elle peut aisément faire sienne, à coup d'injonctions enthousiastes («La vérité! La Nature! Les anciens! Sophocle! Philoctète »). Est-ce la raison pour laquelle il faudra attendre le drame romantique pour retrouver cette énergie antique tant prônée, mais peu appliquée, faute des sujets, de la langue ou du ton pour la soutenir? Gualtiero Calboli, de son côté, évoque les jeux érudits auxquels Diderot se livre dans son essai Sur Térence, mais aussi les jeux de miroir que suscite, comme son ouvrage sur Sénèque, une telle entreprise ( L'Essai Sur Térence de Diderot et la Vie de Térence chez Donat. Étude critique et comparée »). Marilina Gianico revient encore une fois sur le théâtre de Diderot, pour montrer brillamment comment pour Diderot l'Antiquité classique, selon une vision an-historique légitimée par une conception fluide de la Nature et du temps, fonctionne comme un réservoir naturel de formes, de mythes et de techniques d'autant mieux adaptés à notre époque que, laissés longtemps dans l'oubli, ils en retirent une fraîcheur et une émotion nouvelle (“ "Avonsnous eu plus de délicatesse et plus de génie que les Athéniens?" L’Antiquité classique, légitimation de la réforme théâtrale »).

La quatrième et dernière partie (“ L'impact des auteurs anciens sur la pensée de Diderot») regroupe trois contributeurs se signalant par l'originalité de leur approche. Houda Landolsi, dans “Suzanne Simonin, une héroïne de l'Antiquité? ", remarque les points communs, sur le plan théâtral, entre le personnage deLa Religieuse de Diderot et de grandes héroïnes ou héros de l'Antiquité, tels Iphigénie ou Edipe, ou encore le motif $\mathrm{du}$ sacrifice d'un personnage innocent, qui font du roman diderotien une œuvre tragique sans tragédie (contrairement aux "tragédies sans tragique» que l'on trouve au $\mathrm{XvIII}^{\mathrm{e}}$ siècle, époque qui voit l'abandon de cette forme). Aude Lehmann, de son côté, s'empare de la figure de Cicéron dont la présence, moins facilement repérable dans l'œuvre de l'Encyclopédiste que celle de Sénèque (depuis sa Correspondance jusqu'aux Notes écrites de la main d'un souverain aux marges de Tacite), a souvent évoqué un modèle rhétorique de premier plan, ainsi qu'un idéal politique de mesure et d'équilibre républicain (“L'image de Cicéron dans l'œuvre de Diderot»). Enfin Aurélien Gautherie, seul avec Robert Bedon à s'attaquer à l'imposant massif de l'Encyclopédie, traite de la présence de "Celse et le De Medicina", montrant comment les encyclopédistes ont trouvé d'autant plus d'intérêt à la somme médicale latine qu'elle représentait déjà à son époque un projet encyclopédique de grande ampleur, demeuré hélas à l'état de fragments. Diderot, lui-même traducteur du Medical Dictionary de Robert James, ne pouvait rester insensible à une telle entreprise.

Cet ensemble témoigne du très riche et louable effort des antiquisants (parfois non diderotistes) pour mettre à disposition de tous des analyses permettant de mieux apprécier l'œuvre du philosophe - avec un petit regret pour l'absence d'Épicure ou de Lucrèce, dont le matérialiste Diderot a pourtant tiré toute la matière que l'on sait. On soulignera pour finir la qualité des index, rarement d'une telle précision, distinguant les auteurs anciens des auteurs classiques et modernes, les personnages réels et fictifs, les divinités et héros, les lieux et monuments, enfin les notions. Le volume se clôt sur d'utiles résumés en français des contributions.

Odile Richard-Pauchet

Université de Limoges odile.pauchet@unilim.fr 
Élise Lenoux, Mythologie de papier. Donner à voir l'Antiquité entre France et Allemagne (xVIII siècle-milieu du XIX ${ }^{e}$ siècle), préface de François Lissarrague, Dijon, Éditions Universitaires de Dijon, 20I8, 36ı p., 25 €/ ISBN 978236441275o.

Dans cet ouvrage de 36r pages issu de sa thèse soutenue en 2015 et dirigée par François Lissarrague à l'École des Hautes Études en Sciences Sociales, Élise Lehoux (EL) a cherché à retracer l'histoire de la mythologie figurée en France et en Allemagne, du xvIII siècle au $\mathrm{XIX}^{\mathrm{e}}$ siècle, période plutôt négligée par l'historiographie. Pour ce faire, elle a croisé plusieurs disciplines : histoire des religions, histoire du livre, histoire des techniques de reproduction, histoire de l'enseignement.

Son étude se compose de trois parties. Débutant par l'ère des Antiquaires et leurs mythologies illustrées, elle se poursuit par la présentation des premiers livres d'images sur la mythologie qui paraissent en Allemagne puis en France. Elle s'achève avec l'histoire des deux genres majeurs du livre archéologique : les monuments et les recueils spécialisés sur la céramique. Le lecteur est conduit à comprendre la constitution d'un savoir mythologique dans le cadre de la professionnalisation de l'archéologie, entre ı820 et I85o. Pour l'auteure, en effet, “les mythologies de papier » doivent être étudiées comme des laboratoires donnant accès à une forme de connaissance ainsi qu'à sa transmission à différentes échelles. La mise en parallèle des savants de la France et de l'Allemagne souligne la différence des cadres institutionnels entre les deux pays.

Dans la première partie qui traite de l'émergence de la mythologie figurée (p. 2I-Iog), l'auteure s'interroge sur l'apport de trois personnalités majeures pour son sujet. Si Bernard de Montfaucon est l'“ ancêtre fondateur » d'une nouvelle interprétation des images, le comte de Caylus est présenté comme l'innovateur et Johann
Joachim Winckelmann comme l'inventeur d'une nouvelle discipline : l'histoire de l'art. C'est moins la biographie de ces personnalités qui nourrit l'intérêt de l'historienne que leur méthode d'analyse des images, leur réseau d'informateurs en Europe et leur place dans la construction du savoir mythologique par l'image et les textes. Le second chapitre de cette partie prend en compte l'apport pédagogique des images dans les livres illustrés. Alors qu'en France, il s'agit de former les jeunes gens à une culture élitiste, en Allemagne, un large lectorat est recherché par les premiers ouvrages archéologiques illustrés. Pour autant, ces “ musées de papier » ne sont pas seulement des manuels à vocation pédagogique. S'ils cherchent autant à instruire qu'à séduire, ils ont aussi pour finalité de conserver, d'analyser et de diffuser des connaissances en colligeant tous les supports: vases, sculptures, monnaies, gemmes.

La seconde partie, riche de trois chapitres, porte sur deux notions: la formalisation et l'uniformisation des savoirs sur la mythologie, dans les années I8oo-I820 (p. II323o). Dans sa présentation des Bildbücher sur la mythologie, EL souligne l'évolution de ces livres d'images tant pour leur forme que pour leur apport à différents publics en Allemagne (I80o-I820), en France (I80o-I85o) et en Europe, entre i820 et Igoo. Comme dans la partie précédente, les personnalités sont les jalons de la généalogie des mythologies de papier. À tous, elle applique la même méthode : éléments biographiques, objectifs recherchés et analyse de l'image. Elle souligne l'originalité du «moment Creuzer » du nom de cet archéologue qui considéra la mythologie antique et figurée comme un langage symbolique. Traduit en français par Joseph-Daniel Guigniaut, son œuvre eut une grande influence en Europe. Le second chapitre est un hommage rendu à Aubin-Louis Millin, premier professeur d'archéologie en France, présenté comme un des derniers polymathes de l'époque moderne. Sa Galerie mythologique, dont est 
relatée la genèse et analysée la structure, fut publiée dans un petit format. Tout en rappelant par son titre le lieu d'exposition des œuvres d'art, ce livre est novateur par l'insertion de récits mythologiques. Dans le troisième chapitre de cette partie, l'auteure présente de façon détaillée le premier manuel de mythologie figurée, le Handbuch composé par K. O. Müller, livre dont le succès fut européen. JeanAntoine Letronne en a souligné la qualité et l'intérêt en félicitant son auteur : “Vous êtes, Monsieur, du petit nombre de ceux qui unissent la science des mots à celle des choses. »

Loin d'être une énumération fastidieuse de tous les savants qui ont œuvré à la constitution d'un savoir archéologique fondé sur l'étude des images mythologiques, ce livre séduit par la finesse de ses analyses, la qualité de ses illustrations et la clarté de son exposition. Il raconte comment d'esthétique, l'image mythologique est devenue un document iconographique. Dans ce type d'essai, les figures les plus fortes pourraient souffrir d'une esquisse qui les rangerait à la suite d'autres, plus mineures. Mais l'auteure ne tombe pas dans ce travers. Ses portraits sont hiérarchisés et les influences mutuelles sont soulignées tout au long de la démonstration.

De plus, EL accorde une large place aux graveurs et aux peintres qui ont fait de ces monuments de papier des œuvres d'art par la qualité de leurs dessins ou peintures. C'est là un des intérêts de son enquête que l'attention portée à la technique de reproduction des images. Il y eut la gravure au trait aussi bien sur métal, sur bois qu'en lithographie, réalisée par John Flaxman (I755-1826), puis la “machine Collas», le pantographe en trois dimensions inventée en I836 qui permet de réduire des basreliefs de grandes dimensions. Quant à la prestigieuse collection des vases grecs réunis par l'ambassadeur et collectionneur anglais Hamilton, elle est composée de i8o images qui sont de véritables tableaux. EL ne se contente donc pas d'une approche descriptive, elle ne néglige ni la mise en page, ni les jeux graphiques, ni les montages opérés pour donner sens aux images et orienter le lecteur. Elle souligne ainsi que l'évolution des techniques contribue à l'évolution de l'esthétique.

Une bibliographie complète cet ouvrage de référence pour tous ceux qui s'intéressent aux origines de l'archéologie et au statut de la représentation dans l'étude des religions.

\section{Geneviève Hoffmann \\ Université de Picardie Jules Verne genevieve.hoffmann@orange.fr}

\begin{abstract}
Dino Piovan, Tucidide in Europa. Storicie storiografia greca nell'età dello storicismo, postface de Ugo Fantasia, Milan et Udine, Mimesis Edizioni, 20I8, I8o p., ı 8 / ISBN 9788857550602 .
\end{abstract}

Il libro di Dino Piovan, accompagnato da una stimolante postfazione di Ugo Fantasia, è articolato in quattro capitoli, di cui tre già pubblicati come saggi autonomi, ognuno dei quali seguito da un ricco post-scriptum con aggiornamenti bibliografici. Il volume ha il notevole merito di ricostruire la fervida stagione di studi tucididei gravitanti attorno al magistero di Gaetano De Sanctis tra le due guerre mondiali, discutendola in rapporto agli indirizzi esegetici di area tedesca e, soprattutto, nel quadro della cultura politico-filosofica italiana del primo Novecento, dominata da un lato dal consolidamento del regime fascista e, dall'altro, dallo storicismo idealista di Benedetto Croce.

Il capitolo I, Tucidide in Germania: tra storicismo e filologia, costituisce "una premessa necessaria sul piano storiografico » (p. I7) all'intera monografia. Qui, Piovan ripercorre il consolidamento del "culto ottocentesco di Tucidide» 
(l'espressione è di Arnaldo Momigliano) all'interno della tradizione storicista germanica, approfondendo gli scritti di Leopold von Ranke e rintracciandovi la canonizzazione di un'immagine idealizzata di Tucidide come padre fondatore della moderna storiografia scientifica e, insieme, maestro di prosa narrativa. Sul versante opposto, l'autore illustra i principali sviluppi della questione tucididea (Thukydideische Frage), che, aperta da Franz Ullrich nel I846, minacciava di compromettere l'unità ideologica e artistica delle Storie, minando così, alle fondamenta, il « culto tucidideo ». Degna di nota, al riguardo, è la discussione delle posizioni unitarie di Eduard Meyer e l'agile presentazione dell'opzione disgregante di Eduard Schwartz.

Nella restante parte del libro, Piovan approfondisce la riflessione su Tucidide elaborata da De Sanctis e dai suoi allievi Aldo Ferrabino e Arnaldo Momigliano -, raccordandola con la tradizione di studi tedesca e con il più ampio dibattito sul significato complessivo della storia greca animato dalla Griechische Geschichte di Karl Julius Beloch. Il capitolo II (Gaetano De Sanctiso Tucidide critico dell'impero) rivela la complessità delle posizioni del caposcuola. Da un lato, è percepibile in De Sanctis il rifiuto di ogni esaltazione incondizionata di Tucidide, ben evidente nelle critiche mosse all'Impero atheniese di Ferrabino (1927), colpevole di un atteggiamento oltremodo passivo nei riguardi del testo tucidideo. Dall'altro lato, tali giudizi erano temperati dal riconoscimento della grandezza dell'autore ateniese, sottolineata a più riprese nel capitolo a lui dedicato nella Storia dei Greci (I939). Quanto alla componente crociana, essa emergeva soprattutto nel trattamento della Thukydideische Frage, laddove De Sanctis - risentendo dell'approccio dell'allievo Momigliano - approfondiva l'evoluzione della storiografia tucididea in termini di "svolgimento di pensiero", valutando i problemi della stratigrafia compositiva in funzione della «formazione del pensiero storico» (p. 70-7I). La caratterizzazione di un Tucidide critico dell'impero - delineata a partire dal Dialogo dei Meli - pareva inoltre inserirsi all'interno di una più generale visione etico-politica che mostrava punti di contatto con la concezione crociana della storia come storia della libertà. Tutta desanctisiana - e legata al suo intenso cattolicesimo - era invece l'individuazione, nel contesto del medesimo dialogo, di un genuino senso tucidideo del divino, non privo di coloriture provvidenzialistiche.

L'interpretazione di Ferrabino, illustrata nel capitolo III (Un moderno interprete tucidideo: Aldo Ferrabino), si muoveva su linee completamente diverse, ma rivelava lo stesso intreccio di influenze eterogenee. Il trattamento di Tucidide nell'Impero atheniese implicava - come detto - un'adesione strettissima al resoconto antico, che portava Ferrabino a svalutare pregiudizialmente testimonianze alternative a quella delle Storie e a prescindere da gran parte della bibliografia moderna. Tale impostazione, che si sorreggeva sulla convinzione che l'opera tucididea fosse pienamente credibile, venne ribadita in un corso universitario di pochi anni successivo-Alcibiade. Esegesi di Tucidide (I93I) -, nel quale l'argomentazione ferrabiniana assumeva toni apologetici. Questo atteggiamento non era tuttavia sufficiente a fare di Ferrabino un " teologo » di Tucidide, « come allora venivano definiti i sostenitori incondizionati dello storico ateniese » (p. 78). Lo studioso - osserva Piovan - non si accontentava di fondare la propria lettura della realtà antica sulla sola parola tucididea, ma approdava a un'interpretazione del tutto personale e modernizzante della storia greca che spiegava l'evoluzione politica dell'Ellade alla luce del criterio “ben novecentesco» - «dello Stato forte come Stato forte » (p. 84), rivelando in ciò chiare convergenze con l'ideologia fascista e con l'attualismo gentiliano.

Il quarto e ultimo capitolo (Tucidide, Momigliano e lo storicismo) prende le mosse 
dalla tesi di laurea di Arnaldo Momigliano - La composizione della storia di Tucidide -, discussa a Torino nel ig29 sotto la guida di De Sanctis. Il lavoro, pubblicato nel I93o, ha nell'Impero atheniese di Ferrabino un antecedente diretto, la cui influenza emerge nella fascinazione di un Tucidide scopritore della legge universale della " forza come motore della storia » (p. Io3). Ciononostante, l'opera ferrabiniana costituiva solo il punto di partenza di un'analisi in realtà più complessa, che si riallacciava da un lato allo studio filologico della questione tucididea e, dall'altro, mostrava evidenti debiti nei confronti dello storicismo crociano, declinati lungo tre direttrici principali: I) i rapporti con la storiografia di Erodoto, rispetto alla quale Tucidide costituiva un “superamento » (p. Iо5); 2) l'aspirazione a unificare pensiero storico e filosofia, tangibile nella convinzione che le Storie potessero spiegarsi solo all'interno del quadro intellettuale definito dalla sofistica; 3) un approccio alla Thukydideische Frage che trasformava - anticipando De Sanctis - la questione filologica in problema di pensiero. Nonostante l'influsso crociano, permanevano però, almeno nel primo Momigliano, tracce della tradizione rankiana, evidenti nell'esaltazione dell'opera di Tucidide come imperituro capolavoro storiografico. Proprio questi riferimenti all'esemplarità tucididea iniziarono a diradarsi a mano a mano che il debito con la filosofia di Croce si approfondiva e Momigliano andava elaborando, all'interno della monografia Filippo il Macedone (I934), uno schema di sviluppo storico nel quale “ civiltà greca, ellenistica, giudaica, romana venivano a confluire positivamente e a riannodarsi, attraverso il Cristianesimo, con la storia e la coscienza moderne » (p. Io8). In questa lettura ad ampio raggio del mondo antico, il ruolo di Tucidide risultava sensibilmente ridimensionato e la sua opera veniva interpretata come espressione di una fase storica - quella di V secolo - destinata a essere sopravanzata dal progressivo incedere delle epoche successive.

Al termine del suo complesso itinerario, l'analisi di Piovan permette insomma di chiarire, oltre ai variegati rapporti instaurati dalla scuola desanctisiana con la tradizione esegetica di area tedesca, gli stretti legami intrattenuti da De Sanctis, Ferrabino e Momigliano con il panorama politicofilosofico dell'Italia primonovecentesca. In tale ottica, com'era negli auspici dell'autore, la ricezione di Tucidide si rivela « una sorta di prisma » (p. I8) utile per ripensare la storia dell'antichistica italiana tra le due guerre come parte di una vicenda culturale più ampia, segnata dalla dialettica con la filosofia neoidealista e con il clima, oppressivo e autoritario, imposto dal fascismo.

Luca Iori

Università di Parma luca.iori@unipr.it
Sylvie Rougier-Blanc (éd.), Athénée de Naucratis. Le banquet des savants, livre XIV : Spectacles, chansons, danses, musiques et desserts (texte, traduction et notes - études et travaux), Bordeaux, Ausonius, 20I8, 8I2 p. (2 vol.), $45 € /$ ISBN 9782356132369 .

L'avant-propos du premier volume revient sur l'origine, le parcours du projet et le choix du livre XIV. Une notice présente les choix éditoriaux. Le texte grec est présenté dans une forme proche de sa version manuscrite, accompagné d'un apparat critique complet, tandis que la traduction propose une typographie ergonomique facilitant l'identification des citations et des différents niveaux de lecture. Le texte français est présenté au regard du contenu par un découpage moderne: Vin et folie ; Bouffons amateurs de plaisanteries ; Auloi et chansons; Rhapsodes et artistes de banquet ou de scène; la musique et son 
rôle ; Les danses ; Retour à la musique ; Les instruments de musique; Les deuxièmes tables; Catalogue des gâteaux; Les fruits ; Viandes et volailles; Les fromages; Propose d'un cuisinier sur les mageiroi et sur le myma; Recherche sur la mattyê. Les notes complémentaires sur la traduction sont renvoyées, lorsqu'elles sont denses, en fin de texte, occupant près de la moitié du volume (p. 2I7-387). Elles sont suivies d'une liste des auteurs cités par Athénée et d'un dossier iconographique sur les instruments de musique.

Le second volume consacré aux études s'articule en trois parties: tout d'abord un important travail de Jean-Claude Carrière autour d'Athénée et de son œuvre. Un premier temps est consacré au portrait de l'auteur, à son origine, à sa formation et son fonctionnement vis-à-vis de ses contemporains. Les personnages du cercle de Larensios sont passés en revue, entre éléments d'historicité et constructions littéraires. Ce sont ensuite les indices de nature mémorielle ou historique qui sont jaugés avec prudence, en rappelant la fragilité de toute hypothèse construite à partir de ces éléments. Les mentions d'empereurs romains sont examinées avec attention tout comme les éléments faisant l'éloge de Rome, puis l'œuvre d'Athénée est contextualisée au regard des mouvements intellectuels, politiques et culturels qui ont cours dans l'empire aux II $^{\mathrm{e}}$ et $\mathrm{III}^{\mathrm{e}}$ siècles. Le positionnement d'Athénée vis-à-vis des auteurs anciens mais aussi de ses contemporains, jamais mentionnés explicitement, est également analysé afin d'éclairer son projet et son ton d'écriture. En dernier lieu, le parcours personnel d'Athénée et sa trajectoire depuis Naucratis et Alexandrie vers Rome sont envisagés, ainsi que l'image qu'il donne de ces trois cités. Deux appendices clôturent cette partie. Le premier liste les mentions de Séleucides dans l'œuvre. Le second évoque les problèmes de composition de l'ouvrage et questionne notamment les dif- férents niveaux et temporalités de lecture possibles.

La deuxième partie du tome 2 interroge la méthodologie athénéenne dans le livre XIV. Sylvie Rougier-Blanc compare l'usage d'Homère et des poètes archaïques (Archiloque, Alcée, Pindare, Alcman, Anacréon, Stésichore, Bacchyle, Solon, Hipponax, Simonide, Sappho, Hésiode) dans les citations d'Athénée. Malgré son statut, Homère subit les mêmes déformations et manipulations que les autres auteurs qui servent eux tantôt de caution chronologique, d'élément d'autorité ou de ressource lexicologique. Sa relecture par Athénée, qui pourrait sembler plus respectueuse, n'est pourtant pas dénuée d'ironie. Benoît Louyest analyse ensuite le choix formel d'Athénée qui imite la construction scientifique des lexika de son temps tout en les tournant en ridicule. Il identifie ainsi plusieurs mécanismes permettant de voir dans l'architecture athénéenne une écriture parodique. In fine le Naucratite développe une écriture acrobatique et réflexive jonglant entre imitation et dérision.

La troisième partie, intitulée « morceaux choisis ", regroupe quatre analyses thématiques. Valérie Visa-Ondarçuhu montre que l'association de la pyrrhique aux Lacédémoniens relève d'un choix de la part d'Athénée, tout comme son récit de l'origine et de l'évolution de cette danse guerrière. Confrontant l'auteur aux autres sources disponibles elle met en lumière la subjectivité de l'auteur et sa volonté de dépeindre une Sparte guerrière et traditionnelle par opposition au reste de la Grèce. Alain Ballabriga aborde la question de la représentation des défilés phalliques et des railleries rituelles chez Athénée, notamment à travers la citation de Sémos de Délos. Le Naucratite propose une digression historique montrant l'hostilité à l'égard de ces railleries qu'il lie au destin politique des libertés. In fine il cherche à donner au lecteur une vision 
bariolée des comédies hellénistiques, entre pièces assagies et reliquats archaïques moqueurs, entre Ancienne et Nouvelle Comédie. Luciana Romeri revient, à travers la fonction du mageiros et le commentaire d'Athénion, sur la question de l'origine et des vertus civilisatrices de l'art culinaire. Athénée fait dialoguer à ce sujet Platon, Athénion et Aristophane, présentant des points de vue opposés sur la signification de la présence dans les cités du cuisinier, personnage cultivé mais non doté d'un métier spécialisé dans la cité idéale. JeanMarc Luce propose un relevé lexical de 82 entrées, pour seulement 3 recettes, des gâteaux chez Athénée. Il compare ensuite cette mise en scène du savoir aux autres projets lexicographiques de l'époque avant de dresser le cadre chronologique et géographique de ces citations démontrant un grand effet de variatio. En dernier lieu, l'auteur interroge la question de l'immoralité fondamentale de la pâtisserie proposée par Athénée. Une bibliographie générale clôt le volume, distinguant sources anciennes (p. $75 \mathrm{I}^{-776}$ ) et études modernes (p. 777-8o6).

Cette publication, fruit d'un travail de longue haleine, remplit son double objectif. D'une part elle fournit un texte grec du livre XIV qui respecte toute la scientificité qu'on peut attendre d'une édition critique tout en livrant une traduction en français originale et accessible. Le second volet, débordant largement du seul livre XIV, atteint son but de montrer Athénée et son œuvre sous un autre jour, infiniment plus complexe que celui de simple compilateur qui prévaut parfois encore. Les études thématiques n'épuisent pas les angles d'approche mais ouvrent la voie à de nouveaux travaux et offrent de nouvelles clefs de lectures enthousiasmantes.

Arnaud Saura-Ziegelmeyer Université Toulouse Jean Jaurès a.sauraziegelmeyer@gmail.com
Isabelle TorRance, Euripides, Londres et

New York, I. B. Tauris, 20I9, I82 p., \$20.65 / ISBN 978I848856684.

Cet ouvrage, qu'Isabelle Torrance propose comme une introduction à l'œuvre d'Euripide, offre une synthèse concise des spécificités littéraires et dramatiques du théâtre du poète. Organisée en six chapitres thématique qui peuvent être abordés indépendamment les uns des autres, cette introduction, nourrie d'exemples précis et développés, et structurée de façon claire constitue une entrée complète et bien illustrée à l'œuvre du poète tragique. Si rien à première vue ne le suggère, la réception d'Euripide (pour la majeure partie des réécritures et adaptations de l'époque moderne et contemporaine) est fréquemment convoquée pour illustrer le propos ou prolonger la réflexion.

Le premier chapitre introduit le théâtre grec et se centre peu à peu sur Euripide. L'auteure présente de façon brève la vie d'Euripide, développe le contexte de représentation des tragédies lors des compétitions tragiques, ses critères religieux, politiques et stylistiques. La présentation de l'histoire de la transmission textuelle d'Euripide mène aux enjeux de l'attribution des pièces, de l'établissement du texte, des fragments et des pièces perdues. En dernière partie de chapitre la présentation des catégories aristotéliciennes sur le dénouement souligne les innovations d'Euripide en la matière et l'influence que ce dernier a ainsi eue sur le développement de la comédie et, plus tard, de l'opéra.

Le second chapitre présente les caractéristiques dramatiques "spectaculaires» particulières à Euripide. Les morts de personnage en scène, les épiphanies qui ouvrent et ferment les pièces, les procédés scéniques utilisés pour les représenter, les objets et les costumes, les innovations en matière de musique et de danses contribuaient au caractère spectaculaire de son théâtre. 
Le chapitre Religion and Philosophy défend l'idée que dans la dramaturgie d'Euripide, les actions divines sont indissociables des questions philosophiques. Les intrusions artificielles en scène des dieux au moyen du deus ex machina, contrebalancées par « la complexité de la fabrique philosophique à l'intérieur des pièces » (the complexity of that philosophical fabric) n'apportent pas de résolution propre et ont pour but de faire réfléchir l'audience. Le recours extrêmement fréquent à Euripide chez Platon dans ses dialogues et dans les œuvres philosophiques ultérieures serait aussi la preuve de la nature intellectuellement et philosophiquement stimulante des tragédies d'Euripide pour le public.

Le chapitre 4 étudie la rhétorique dans l'œuvre d'Euripide. Les scènes d'agôn, calquées sur le système légal athénien, ont pour caractéristique chez Euripide de ne jamais aboutir à un accord. Souvent métathéâtrales, elles interrogent le pouvoir de la rhétorique. Les procédés rhétoriques employés participent aussi à la construction dramatique des personnages et de leur identité. Les comédies d'Aristophane font mention de la "sophistication rhétorique » d'Euripide, dont témoignent les nombreuses références à Euripide chez les orateurs analysées en seconde partie de chapitre.

Le chapitre 5 aborde les dimensions méta-poétiques de l'œuvre d'Euripide. Nombreuses sont les scènes qui trouvent leur source dans les poèmes homériques. Certaines de ses pièces, comme Electre ou Oreste, sont construites comme des réponses à des tragédies d'Eschyle. Les références à Sophocle sont, elles, moins fréquentes et évidentes à discerner. Le recours à des références épiques et tragiques sophistiquées est une source d'innovation et lui permet de se situer dans une tradition littéraire. Ces références engagent son audience, qui doit pouvoir les saisir les références pour apprécier la “ sophistication » des œuvres.
Le dernier chapitre illustre trois thématiques euripidéennes majeures au prisme de leur réception: la «bataille des sexes " (battle of the sexes), la confrontation entre les hommes et les dieux et la guerre. L'enjeu des relations homme-femme est illustré dans Hippolyte et Médée. Dans les différentes réécritures et adaptations d'Hippolyte, cet enjeu demeure central jusqu'à L'amour de Phèdre de Sarah Kane en r99o. Sénèque excepté, les réécritures de Médée ont suivi la tendance euripidéenne à faire de Médée une victime de son statut de femme ou d'étrangère. La réception des Bacchantes interroge les rapports entre hommes et dieux. Dans les années I960, la pièce est associée à la libération sexuelle et le conflit entre Penthée et Dionysos interprété comme une réflexion sur les normes sociales, alors que d'autres adaptations privilégient l'aspect rituel et les interrogations politiques soulevées par la tragédie. Enfin Iphigénie à Aulis et Les Phéniciennes font l'objet d'adaptations politiques qui abordent les violences irréparables de la guerre. Les Troyennes sont à maintes reprises lues comme une critique de la guerre et de l'impérialisme et nombreuses sont les adaptations d'Héraclès qui ont lu dans la folie du héros un syndrome post-traumatique dû à son retour de la guerre.

L'étude de la réception d'Euripide souligne judicieusement la permanence des questions sociales et politiques encore soulevées par les tragédies d'Euripide mentionnées en conclusion. Peut-être aurait-on simplement pu attendre que soit nuancé le caractère novateur et radical d'Euripide au regard de la transmission partielle du répertoire tragique qui est présentée au chapitre I. Cependant, le retour fréquent aux notions essentielles et générales du théâtre grec et l'approche intertextuelle choisie dans cet ouvrage soulignent effectivement les particularités d'Euripide, mais enrichissent aussi considérablement le propos, tout en rendant accessible 
l'ouvrage à un large lectorat. La bibliographie finale, récente et fournie, permet l'approfondissement de certaines notions nécessairement abrégées et la densité de certains passages n'efface pas les qualités d'un ouvrage concis qui énonce avec clarté les spécificités du théâtre d'Euripide sans omettre son contexte politique, historique et littéraire immédiat.

Alexia Dedieu

Université Grenoble Alpes alexia.dedieu@univ-grenoble-alpes.fr 